\title{
Six new species of Labiobaetis Novikova \& Kluge (Ephemeroptera: Baetidae) from Madagascar with comments on the validity of the genus
}

\author{
J.-L. Gattolliat ${ }^{1}$
}

Keywords : Ephemeroptera, Baetidae, Labiobaetis, Pseudocloeon, new species, Madagascar.

The genus Labiobaetis recently has been reported from Madagascar by Lugo-Ortiz and McCafferty (1997). The two described species were only known from the nymphal stage. Examination of material from more than 800 samples in Madagascar allows descriptions of six new species: Labiobaetis longicercus, L. dambrensis, L. nigrocercus, L. vulgaris, L. punctatus and $L$. gilliesi. They can be distinguished easily by the shape of the second segment of the labial palp, the shape of the second segment of the maxillary palp, the setation of the labrum, the number of gills, the presence or absence of hindwings and coloration of the abdomen. At present, the imagoes of only five of the eight species are known. They can be identified by the presence or absence of hindwings, coloration of the wings, shape of the sclerotized process between gonopods and shape of the third segment of the gonopods.

As all the species assigned to Labiobaetis were recently reassigned to Pseudocloeon, the status and validity of the genera Labiobaetis and Pseudocloeon are discussed.

Six nouvelles espèces de Labiobaetis Novikova \& Kluge (Ephemeroptera : Baetidae) de Madagascar et commentaires sur la validité du genre

Mots clés : Ephéméroptères, Baetidae, Labiobaetis, Pseudocloeon, espèces nouvelles, Madagascar.

Le genre Labiobaetis a été récemment signalé de Madagascar par Lugo-Ortiz \& McCafferty (1997). Les deux espèces malgaches ne sont décrites qu'au stade nymphal. L'examen de plus de 800 échantillons à Madagascar permet de décrire 6 nouvelles espèces. Elles se distinguent facilement des espèces déjà connues par la forme du deuxième segment du palpe labial, la forme du deuxième segment du palpe maxillaire, la station du labre, le nombre de branchies, la présence ou l'absence d'ailes postérieures et la coloration de l'abdomen. Actuellement, cinq des huit espèces sont connues au stade adulte. Elles se différencient par la présence ou l'absence d'ailes postérieures, la couleur des ailes, la forme du processus sclérifié entre les gonopodes ainsi qụe la forme du troisième segment des gonopodes.

Toutes les espèces du genre Labiobaetis ont été récemment réassignées au genre Pseudocloeon. Le statut problématique et la validité des genres Labiobaetis et Pseudocloeon sont discutés.

\section{Introduction}

Labiobaetis Novikova and Kluge was erected as a subgenus to incorporate species of the Baetis propinquus species group, the $B$. atrebatinus species group and the $B$. molawinensis species group (Novikova \& Kluge 1987). Labiobaetis was raised to generic rank by McCafferty \& Waltz (1995). An attempt to consider

1. Museum of Zoology, P.O. Box 448, CH-1000 Lausanne 17, Switzerland.

E-mail: Jean-Luc.Gattolliat@serac.vd.ch
Labiobaetis as a junior synonym of the much debated genus Pseudocloeon Klapálek was made (Lugo-Ortiz et al. 1999), but the synonymy remains subject to controversy until the larval stage of the type-species $P$. kraepelini Klapálek is known. The status of Pseudocloeon and Labiobaetis are discussed herein.

The genus Labiobaetis sensu McCafferty \& Waltz (1995) has a wide distribution; it appears to be present all over the world except in Australia and Central and South America (McCafferty \&.Waltz 1995). Two new species were described recently from Madagascar (Lugo-Ortiz \& McCafferty 1997). The examination of the material collected by the LRSAE (Laboratoire de Re- 
cherche sur les Systèmes Aquatiques et leur Environnement) allows the description of six new species from the larval stage and for four of them also from the imaginal stage. Reared material permits the association of the imago with the unusual larva of $L$. fabulosus.

The holotypes and part of the paratypes are housed in the Museum of Zoology, Lausanne, Switzerland. Other paratypes are deposited in the Muséum National d'Histoire Naturelle, Paris.

\section{Taxonomy}

\subsection{Labiobaetis longicercus sp. $\mathbf{n}$.}

\section{Nymph}

Maximal length, fully grown female: Body $5.7 \mathrm{~mm}$. Cerci $9.7 \mathrm{~mm}$. Terminal filament $2.2 \mathrm{~mm}$. Fully grown male: Body $4.9 \mathrm{~mm}$. Cerci $7.9 \mathrm{~mm}$. Terminal filament $2.0 \mathrm{~mm}$.

Head. Coloration almost uniformly brown, vermiform marking on vertex and frons, border of sclerites yellow. Antennae light yellow except scapus and pedicellus brown; pedicellus without distolateral process. Turbinate eyes light brown.

Labrum (Fig. 1) rounded, with an anteromedial emargination, dorsally with an arc of about 15 feathered setae, long and thin setae most abundant in middle; distal margin bordered with setae, disto-lateral ones feathered; ventrally with two stout, minute setae and a disto-medial arc of very thin setae.

Right mandible (Fig. 2), stout prostheca apically with rounded denticules; margin between prostheca and mola crenate, without setae, slightly convex; tuft of setae at apex of mola reduced to two small setae; basal half with dorsally long and thin setae.

Left mandible (Fig. 3), stout prostheca apically with denticules and a comb-shape structure; margin between prostheca and mola crenate, almost straight without hump and without setae; tuft of setae at apex of mola absent; basal half with dorsally few long and thin setae.

Hypopharynx (Fig. 4), superlingua with a median projection, covered with black stout setae.

Maxillae (Fig. 5) with 2 small and 2 large teeth, none of them opposed to others; row of setae ending with four long setae, two spine-like setae in middle of row; row of 5 thin setae at basis of galea; one single small seta perpendicular to margin of galea; palp 2segmented, slender, longer than galealacinia, segment 2 with a pronounced distomedial concavity.
Labium (Fig. 6) with glossae clearly shorter than paraglossae; glossae slender; lateral margins with long setae, stouter on inner margin, row of 8 to 9 setae medially; paraglossae stout, apically flattened, with 3 to 4 rows of pectinate setae. Mentum with 5 to 7 thin setae apically. Labial palp 3-segmented slender; first segment elongated with few thin setae; first segment $0.7 x$ length of second and third combined; second segment with a small thumb-like distomedial projection, covered with thin setae and apically with few stouter one, row of 4 long and thin setae dorsally; third segment subconical, inner margin rounded and outer one almost straight, covered with short thin setae and few longer and stouter ones on inner margin.

Thorax. Prothorax brown with large yellow spots. Mesothorax yellow medially, brown with yellow pattern laterally. Metathorax brown with a medial yellow stripe.

Hindwing pads absent.

Legs uniformly light yellow.

Forelegs (Fig. 11), coxa with 3 to 4 stout setae on distal margin.

Femora with dorsally a row of about 10 pointed setae, rare and long in distal part, smaller and more abundant proximally; apicodorsal patch formed by 2 stout and long setae; apex with 3 to 5 short setae and few thin ones; ventral margin with numerous acute setae, longer apically.

Tibiae dorsally with only few thin setae; apically with a single stout pointed seta and a patch of thin setae; ventrally with pointed setae, longer and more abundant apically; tibio-patellar suture present.

Tarsi without seta dorsally; ventral margin with a row of pointed setae increasing in length toward apex; tarsal claws (Fig. 12) with one row of about 11 small teeth; subapical setae absent.

Second and third legs similar to foreleg.

Abdomen. Coloration (Fig. 7) middle to dark brown, lighter laterally, with two central yellow spots, segments 3 and 6 with a yellow central pattern.

Terga (Fig. 9) shagreen with scale bases; posterior margin with blunt spination slightly broader than long.

Sterna light brown with laterally a transverse brown stripe, without scale bases; smooth posterior margin without spines.

Gills (Fig. 8) present on abdominal segments 1 to 7 , tracheation dark brown poorly developed, serrated distally, gill 1 clearly reduced. 

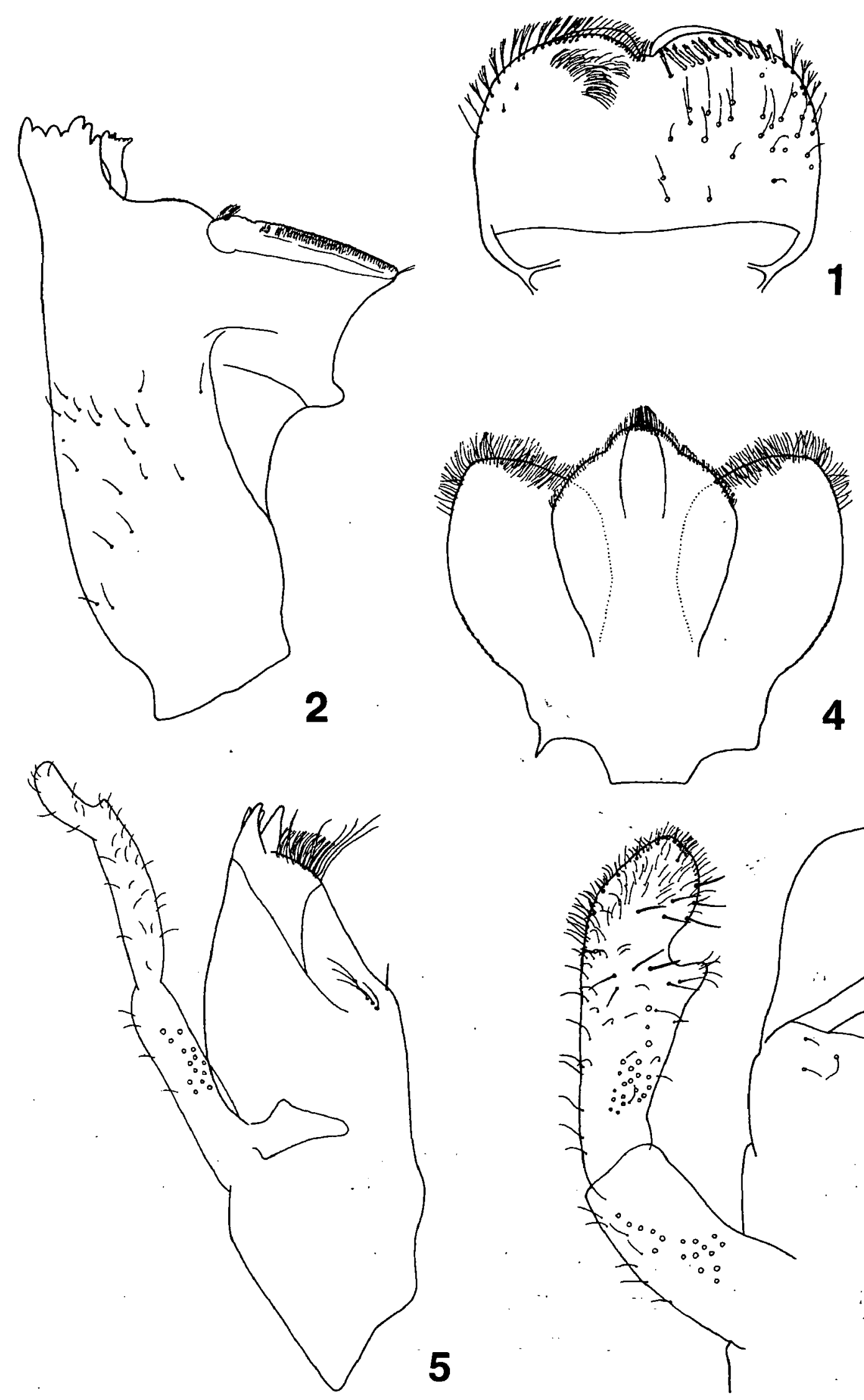

5

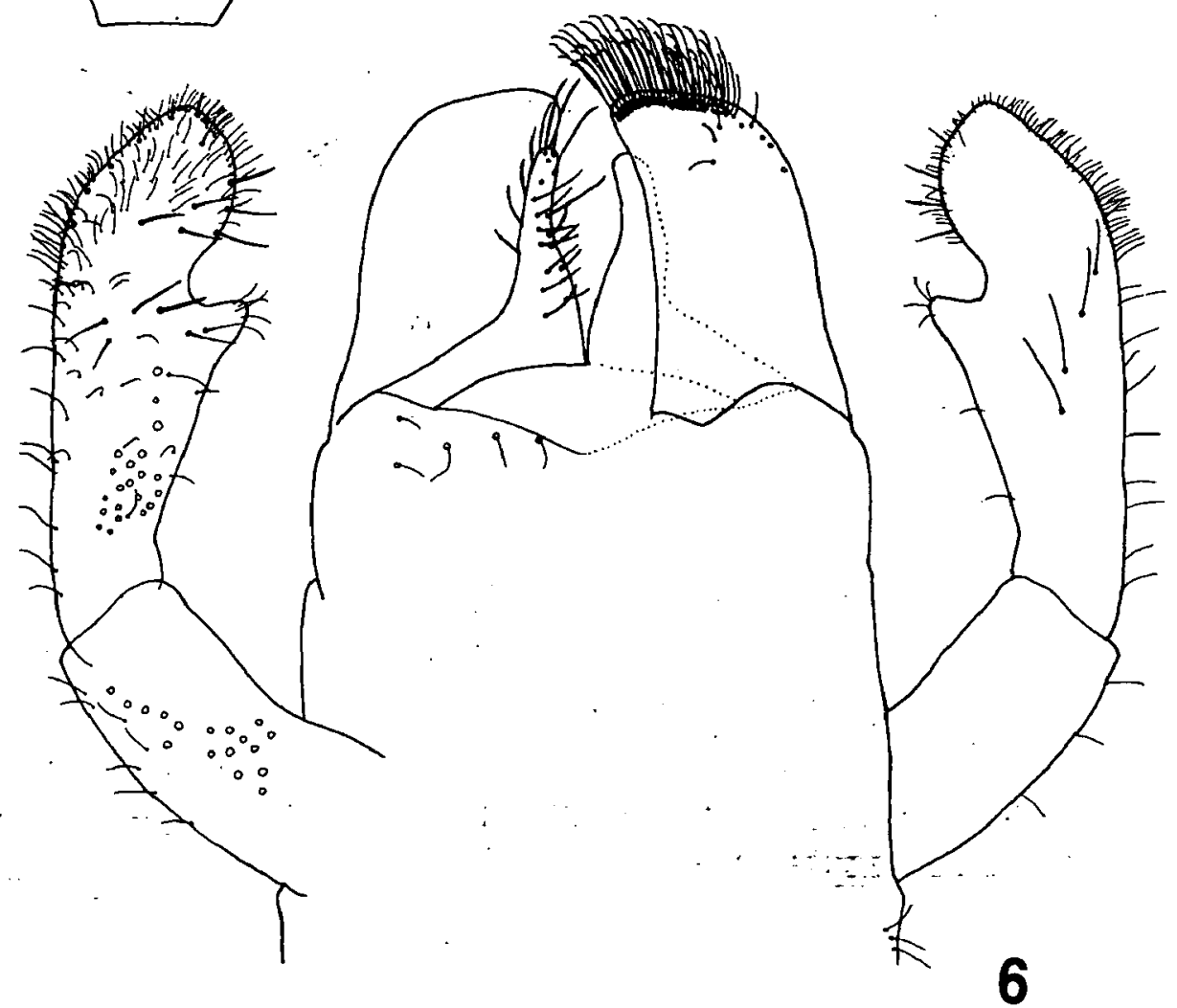

Figs. 1 to 6 . Larval structures of $L$. longicercus sp. n. : $1:$ labrum (left : ventral; right : dorsal). 2 : right mandible. 3 : left mandible. 4 : hypopharynx. 5 : right maxilla. 6 : labium.

Figs. 1 à 6. Structures larvaires de L. longicercus n. sp. : 1 : labre (gauche : ventral; droite : dorsal). 2 : mandibule droite. 3 : mandibule gauche. 4 : hypopharynx. $5:$ maxille droite. $6:$ labium. 


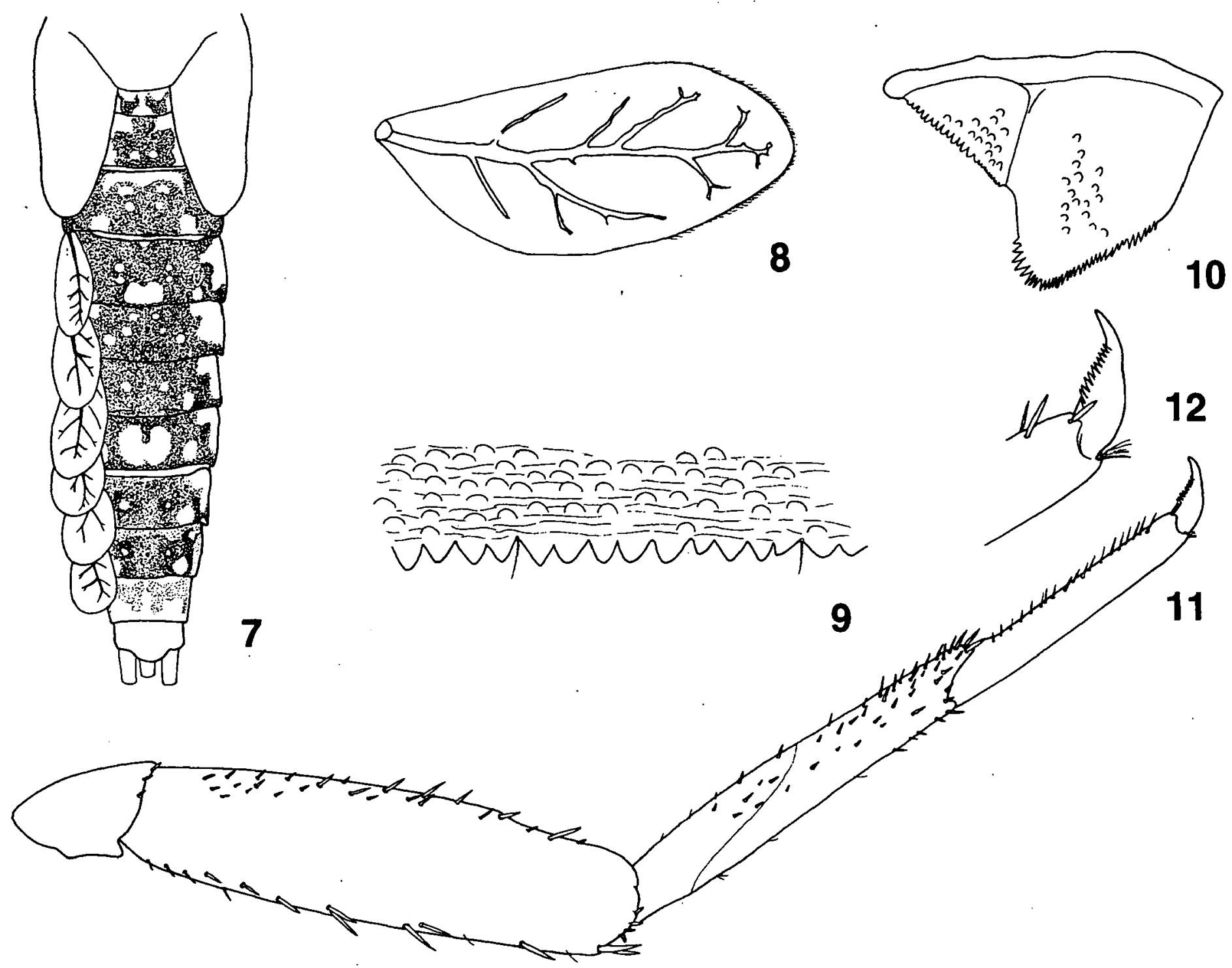

Figs. 7 to 12. Larval structures of $L$. longicercus $n$. sp. : $7:$ abdomen (dorsal view). $8:$ fourth gill. $9:$ detail of tergum. 10 : paraproct. 11 : left foreleg. $12:$ tarsal claw.

Figs. 7 à 12. Structures larvaires de $L$. longicercus n. sp. : $7:$ abdomen (vue dorsale). $8:$ quatrième branchie. $9:$ détail d'un tergite. $10:$ paraprocte. $11:$ première patte gauche. $12:$ griffe tarsale.

Paraproct (Fig. 10) with about 15 scale bases, margin with numerous pointed slender spines; postero-lateral extension with about 13 scale bases, rounded spines along margin.

Cerci coloration yellow, except median segments brown giving a banded appearance.

\section{Adult}

Maximal length, female imago: Body $5.2 \mathrm{~mm}$. Forewing $5.6 \mathrm{~mm}$. Male imago: Body $4.4 \mathrm{~mm}$. Forewing $4.6 \mathrm{~mm}$.

Head. Yellowish brown. Turbinate eyes honey brown with darker basis. Eyes black. Antennae with basis of scapus and pedicellus dark brown, flagellum light yellow with a brown stripe.
Thorax. Forewings $4.6 \mathrm{~mm}$, hyaline with golden brown veins; double intercalary veins between longitudinal veins; pterostigma with 4 to 6 cross-veins generally not reaching subcostal vein (Fig. 13).

Hindwings absent.

Legs uniformly light yellow.

Abdomen. Coloration: female uniformly brown to dark brown; male pale yellow with a dark stripe parallel to distal margin, except segments 7 to 9 brown.

Male genitalia (Fig. 14) with three-segmented gonopods, first and second segments almost fused, first segment without apophysis, abundant thin setae present on inner margin of second segment, third segment globular; well-developed sclerotized process between for- 

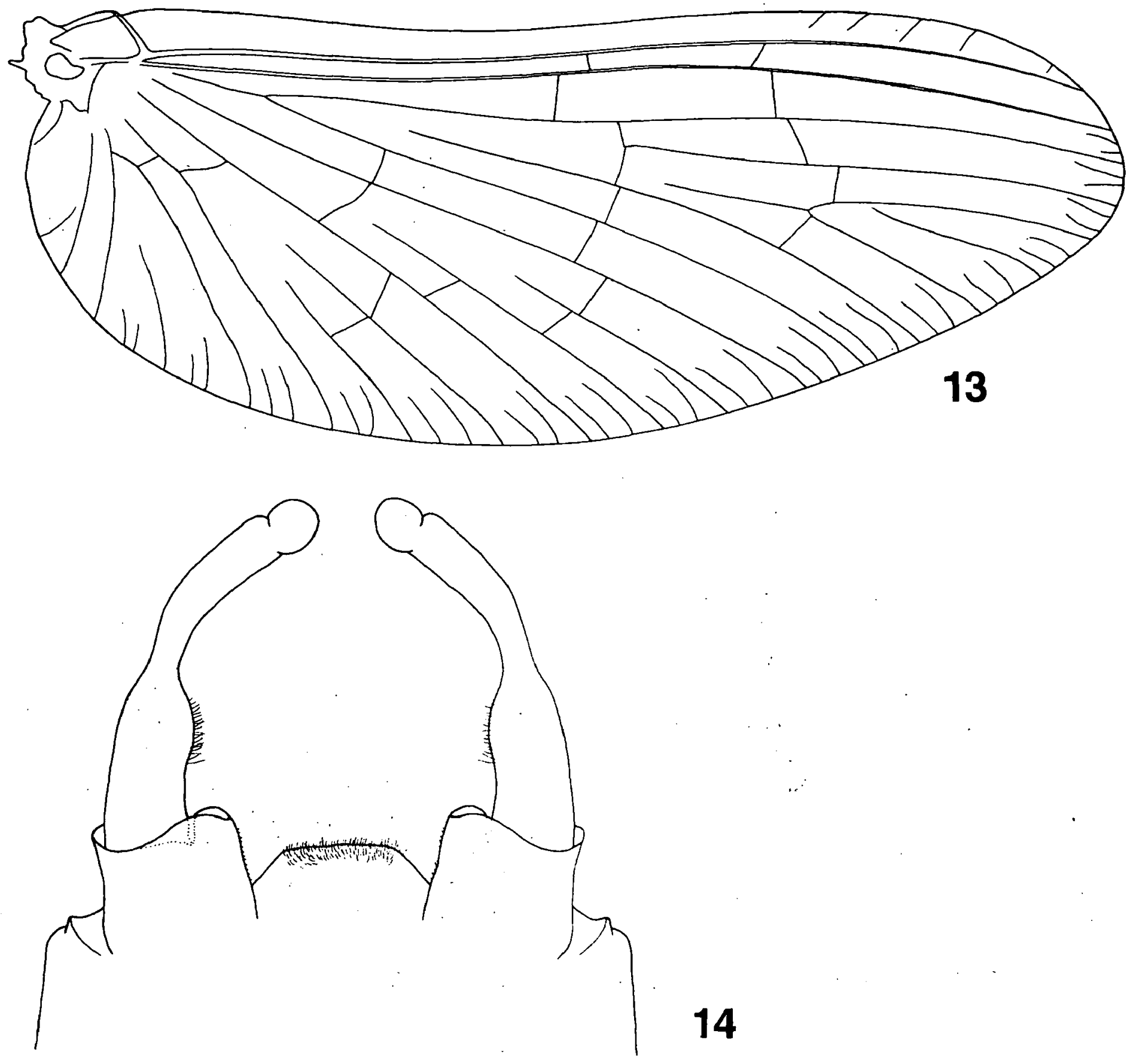

\section{4}

Figs. 13 to 14 . Imaginal male structures of $L$. longicercus sp. n. : 13 : forewing. 14 : genitalia.

Figs. 13 à 14. Structures imaginales males de L. longicercus $n$. sp. : 13 : aile antérieure. 14 : génitalia.

ceps, as broad as distance between forceps, apically flattened, covered with small thin setae.

\section{Material examined}

\section{Holotype}

Male larva, (P0809), Madagascar, Bas. Antongombato, Loc. small dyke near Camp Roussette (Montagne d'Ambre),

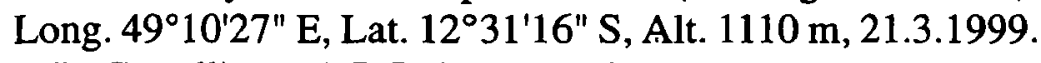
J.-L. Gattolliat and Z. Rabeantoandro.

\section{Paratypes}

81 larvae, male, female imagoes and subimagoes with corresponding larval exuvia, same data as holotype.
74 larvae (P0755), same locality as holotype, 18.3.1999. J.-L. Gattolliat and Z. Rabeantoandro.

Male, female imagoes and subimagoes with corresponding larval exuvia (P0827), same locality as holotype, 25.3.1999. J.-L. Gattolliat and Z. Rabeantoandro.

2 larvae (P0752), Madagascar, Bas. Antongombato, Riv. Makis, Loc. Camp Roussette, Sacred Waterfall (Montagne d'Ambre), Long. $49^{\circ} 10^{\prime} 09^{\prime \prime} \mathrm{E}$, Lat. $12^{\circ} 31^{\prime} 40^{\prime \prime}$ S, Alt. $1075 \mathrm{~m}$, 17.3.1999. J.-L. Gattolliat and Z. Rabeantoandro.

One larva (P0818), same locality as P0752, 23.3.1999. J.L. Gattolliat and Z. Rabeantoandro. 
Larva 819a (on slide), one female subimago with corresponding larval exuvia, same locality as P0752, 25.3.1999. J.L. Gattolliat and Z. Rabeantoandro.

6 larvae (P0825), same locality as P0752, 25.3.1999. J.-L. Gattolliat and Z. Rabeantoandro.

36 larvae (P0753), Madagascar Bas. Antongombato, Loc. small dyke near Camp Roussette (Montagne d'Ambre), Long. $49^{\circ} 10^{\prime} 21^{\prime \prime} \mathrm{E}$, Lat. $12^{\circ} 31^{\prime} 27^{\prime \prime} \mathrm{S}$, Alt. $1100 \mathrm{~m}$, 18.3.1999. J.-L. Gattolliat and Z. Rabeantoandro.

One larva 810c (on slide) and 27 larvae, one male subimago with corresponding larval exuvia and one female subimago with corresponding larval exuvia, Madagascar, Antongombato Bas., Riv. Makis, Loc. Camp Roussette (Montagne d'Ambre), Long. $49^{\circ} 10^{\prime} 21^{\prime \prime}$ E, Lat. 12 $31^{\prime} 27^{\prime \prime}$ S, Alt. $1030 \mathrm{~m}$, 21.3.1999. J.-L. Gattolliat and Z. Rabeantoandro.

Larva 819a (on slide), 32 larvae and one male subimago with corresponding larval exuvia, same locality as P0810, 23.3.1999. J.-L. Gattolliat and Z. Rabeantoandro.

One larva (P0754), Madagascar, Bas. Antongombato, Riv. Makis, Loc. Waterfall of the Antakarana (Montagne d'Ambre), Long. $49^{\circ} 10^{\prime} 20^{\prime \prime} \mathrm{E}$, Lat. $12^{\circ} 31^{\prime} 09^{\prime \prime} \mathrm{S}$, Alt. $1000 \mathrm{~m}$, 18.3.1999. J.-L. Gattolliat and Z. Rabeantoandro.

One larva (P0829), same locality as P0754, 25.3.1999. J.L. Gattolliat and Z. Rabeantoandro.

One larva (P0812), Madagascar, Bas. Antongombato, Riv. Makis, Loc. Great Waterfall (Montagne d'Ambre), Long. $49^{\circ} 10^{\prime} 14^{\prime \prime}$ E, Lat. 12²9'23" S, Alt. 690 m, 22.3.1999. J.-L. Gattolliat and Z. Rabeantoandro.

\section{Etymology}

The specific epithet is Latin expressing the strong development of the cerci.

\subsection{Labiobaetis dambrensis sp. $n$.}

\section{Nymph}

Maximal length, fully grown female: Body $6.2 \mathrm{~mm}$. Cerci $5.8 \mathrm{~mm}$. Terminal filament $2.3 \mathrm{~mm}$. Fully grown male: Body $5.3 \mathrm{~mm}$. Cerci $5.7 \mathrm{~mm}$. Terminal filament $2.2 \mathrm{~mm}$.

Head. Coloration uniformly pale yellowish brown, without vermiform marking on vertex and frons. Antennae pale yellow; pedicellus without distolateral process. Turbinate eyes light brown.

Labrum (Fig. 15) rectangular, distal margin almost straight with a poorly developed anteromedial emargination, dorsally with an arc of about 20 setae not reaching margin, long and thin setae scattered over surface; distal margin bordered with setae, disto-lateral ones feathered; ventrally with three stout, minute setae and a disto-medial arc of very thin setae.

Right mandible (Fig. 16), stout prostheca apically with rounded denticules; margin between prostheca and mola crenate, without setae, slightly convex; tuft of setae at apex of mola reduced to a single seta; basal half slender, dorsally with few long and thin setae.

Left mandible (Fig. 17), stout prostheca apically with denticules and a comb-shape structure; margin between prostheca and mola crenate, almost straight without hump and without setae; tuft of setae at apex of mola absent; basal half slender with dorsally few long and thin setae.

Hypopharynx (Fig. 18), superlingua with a broad median projection, covered with thin setae, apical ones stouter.

Maxillae (Fig. 19) with 2 small and 2 large teeth, none of them opposed to others; row of setae ending with four long setae, two spine-like setae in middle of row; row of 4 long setae at basis of galea; one single seta perpendicular to margin of galea; palp 2-segmented, first part of segment 2 enlarged, with an elongated and pronounced distomedial concavity.

Labium (Fig. 20) with glossae clearly shorter than paraglossae; glossae slender, inner margins with long and stout setae, row of 5 setae medially; paraglossae stout, apically flattened, with 3 to 4 rows of pectinate setae. Labial palp 3-segmented; first segment elongated with few thin setae, subequal in length to second and third combined; second segment with a broad thumb-like distomedial projection laterally rounded, with thin setae, row of 3 long and thin setae dorsally; third segment asymmetrical, inner margin rounded and outer almost straight or slightly concave, covered with numerous short, thin setae, few longer ones on margin and 6 to 8 short stout setae subparallel to outer margin.

Thorax. Coloration pale yellowish brown

Hindwing pads present.

Legs pale yellow, except apex of femora and tibiae slightly darker.

Forelegs (Fig. 24), coxa with a single stout seta on distal margin.

Femora with dorsally a row of about 17 pointed setae, regularly distributed, with patches of 2 to 4 small, thin setae; apicodorsal patch formed by 2 stout, long setae; apex with 5 to 7 short setae and few thin ones; ventral margin with numerous small acute setae, row of small blunt setae subparallel to margin.

Tibiae dorsally with a row of minute setae and few thin setae; apically with a single stout pointed seta and a patch of thin setae; ventrally with small pointed setae, rather regular in length; tibio-patellar suture present. 


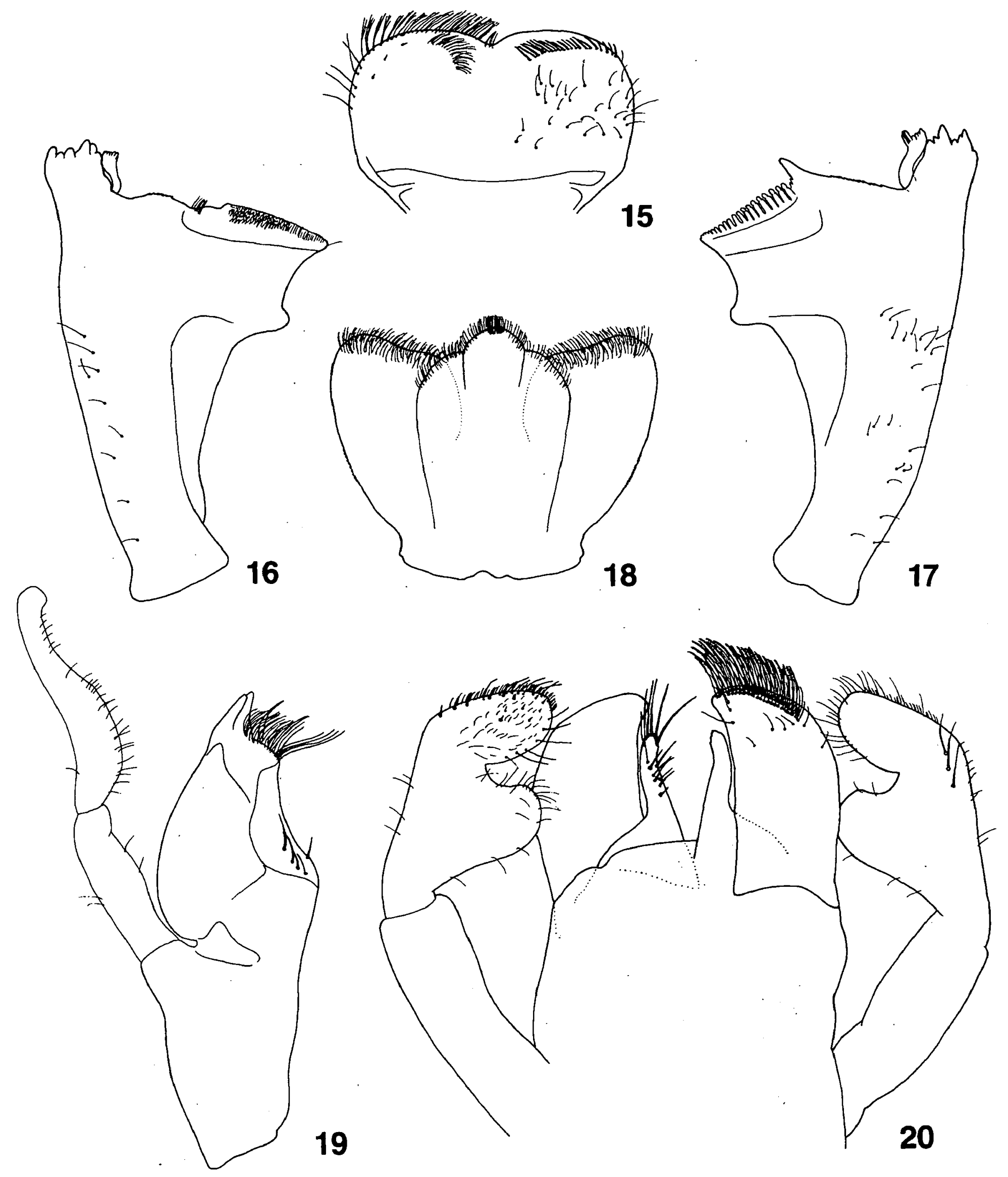

Figs. 15 to 20 . Larval structures of $L$. dambrensis sp. n. : 15 : labrum (left : ventral; right : dorsal). 16 : right mandible. 17 : left mandible. 18 : hypopharynx. 19 : right maxilla. $20:$ labium.

Figs. 15 à 20. Structures larvaires de $L$. dambrensis n. sp. : 15 : labre (gauche : ventral; droite : dorsal). $16:$ mandibule droite. $17:$ mandibule gauche. $18:$ hypopharynx. $19:$ maxille droite. $20:$ labium. 

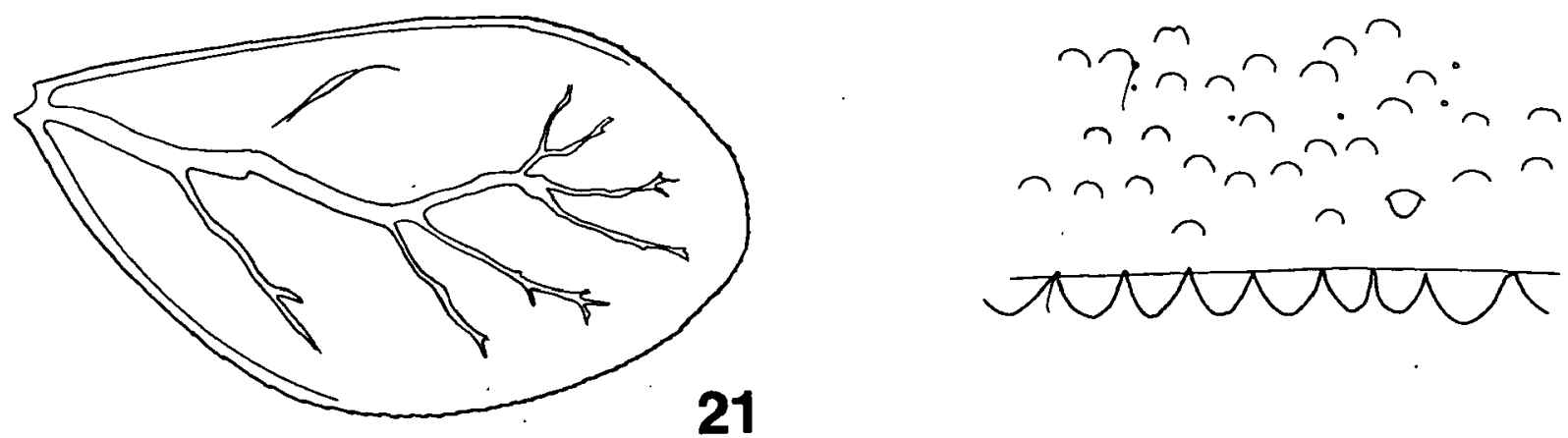

\section{2}

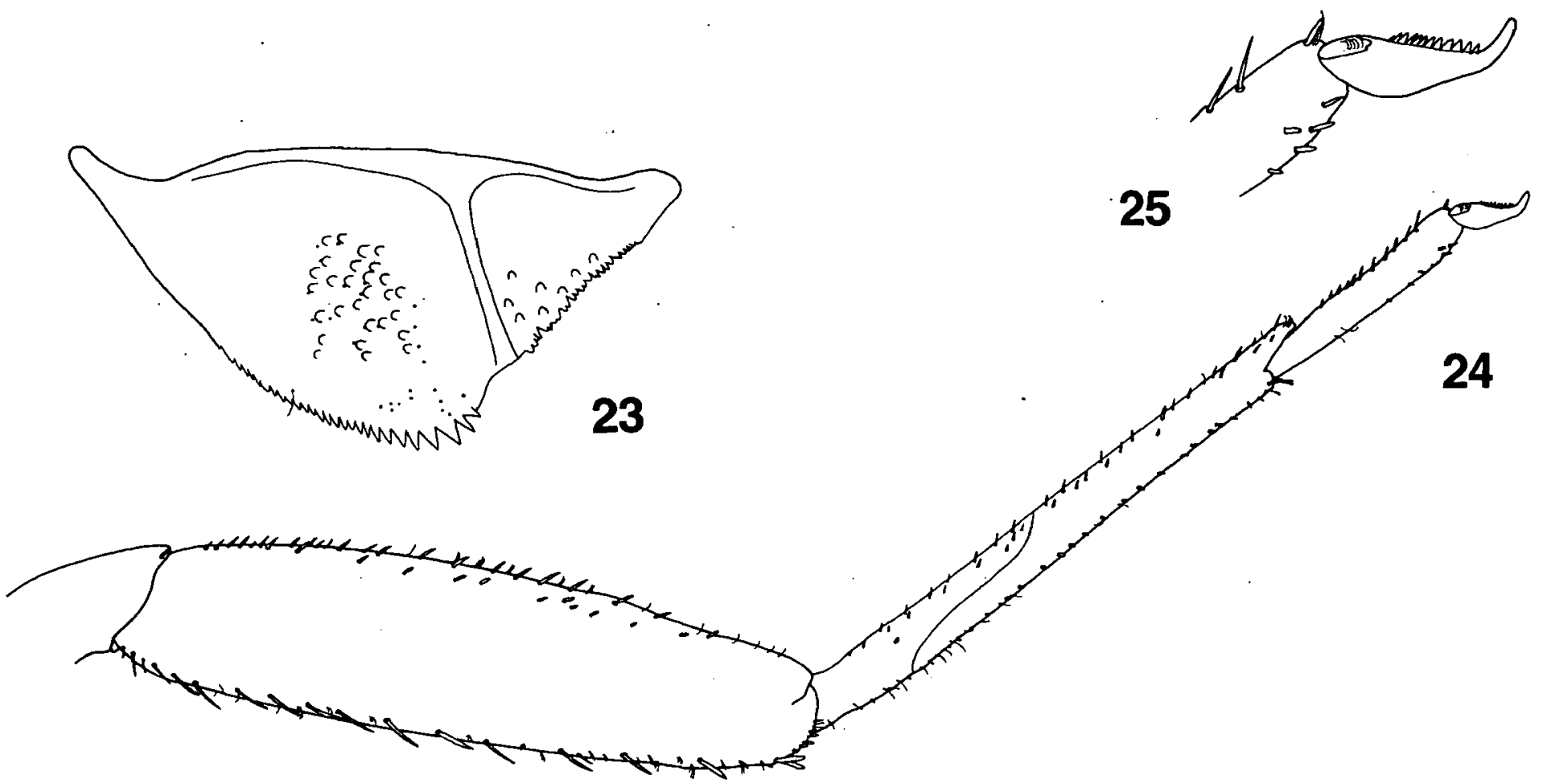

Figs. 21 to 25 . Larval structures of $L$. dambrensis sp. n. : 21 : fourth gill. 22 : detail of tergum. 23 : paraproct. $24:$ left foreleg. 25 : tarsal claw.

Figs. 21 à 25 . Structures larvaires de L. dambrensis n. sp. : 21 : quatrième branchie. 22 : détail d'un tergite. 23 : paraprocte. 24 : première patte gauche. $25:$ griffe tarsale.

Tarsi dorso-apically with small setae; ventral margin with a row of pointed setae increasing in length toward apex; tarsal claws (Fig. 25) with one row of about 9 teeth, proximal ones shortest; subapical setae absent.

Second and third legs similar to foreleg.

Abdomen. Coloration pale yellowish brown, without pattern.

Terga (Fig. 22) weakly shagreen with scale bases; posterior margin with blunt spination generally slightly broader than long.

Sterna pale yellow, shagreen with scales and scale bases; smooth posterior margin without spines.

Gills (Fig. 21) present on abdominal segments 2 to 7 , tracheation dark brown poorly developed, margin brown except distally, serrated distally.
Paraproct (Fig. 23) with about 30 scale bases, margin with numerous pointed spines; postero-lateral extension with about 8 scale bases, pointed spines along margin.

Cerci coloration uniformly yellow.

\section{Adult}

Maximal length, male imago: Body $5.2 \mathrm{~mm}$. Forewing $5.4 \mathrm{~mm}$. Hindwing $0.8 \mathrm{~mm}$.

Head. Pale brown. Turbinate eyes honey brown, and orange brown laterally. Eyes black, generally with a transverse grey stripe. Antennae with basis of scapus and pedicellus dark brown, flagellum yellow.

Thorax. Yellowish brown dorsally, brown ventral- 

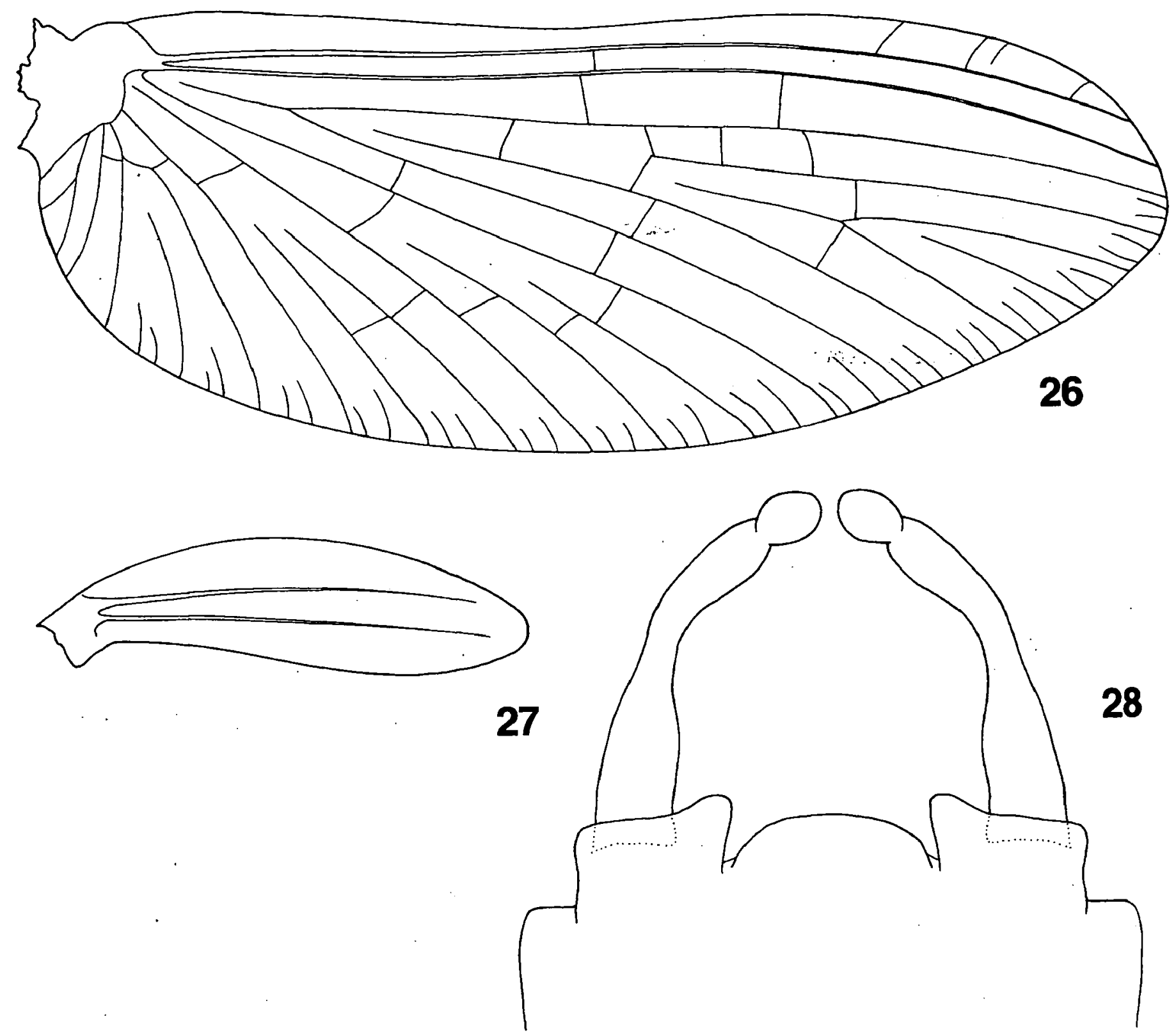

Figs. 26 to 28 . Imaginal male structures of $L$. dambrensis sp. n. : 26 : forewing. 27 : hindwing. $28:$ genitalia.

Figs. 26 à 28. Structures imaginales males de L. dambrensis $n$. sp. : 26 : aile antérieure. 27 : aile postérieure. $28:$ génitalia.

Forewings $5.3 \mathrm{~mm}$, hyaline with hyaline grey veins, except $\mathrm{Sc}$ and $\mathrm{R} 1$ veins golden brown; double intercalary veins between longitudinal veins; pterostigma with 4 cross-veins (Fig. 26).

Hindwings narrow, crescent-shaped, with two longitudinal veins, without costal process (Fig. 27).

Terga pale yellow with darker distal margin; terga 7 to 9 brown.

Sterna hyaline except light brown laterally.

Legs uniformly light yellow.

Male genitalia (Fig. 28) with three-segmented gonopods, first and second segments almost fused, first segment without apophysis, third segment globular; welldeveloped sclerotized process between forceps, as broad as distance between forceps, apically flattened without setae.

\section{Material examined}

\section{Holotype}

Female larva (P0818), Madagascar, Bas. Antongombato, Riv. Makis, Loc. Camp Roussette, Sacred Waterfall (Montagne d'Ambre), Long. $49^{\circ} 10^{\prime} 09^{\prime \prime} \mathrm{E}$, Lat. $12^{\circ} 31^{\prime} 40^{\prime \prime} \mathrm{S}$, Alt $1075 \mathrm{~m}, 23.3 .1999$. J.-L. Gattolliat and Z. Rabeantoandro.

\section{Paratypes}

One female larva $818 \mathrm{~b}$ (on slide), 18 larvae and one male imago, same data as holotype.

2 larvae (P0806), same locality as holotype, 20.3.1999. J.-

L. Gattolliat and Z. Rabeantoandro. 
One male imago $819 \mathrm{~b}$ (on slide) with corresponding larval and subimaginal exuvia and one larva, same locality as holotype, 23.3.1999. J.-L. Gattolliat and Z. Rabeantoandro.

One female larva 825a (on slide) and 6 larvae, same locality as holotype, 25.3.1999. J.-L. Gattolliat and Z. Rabeantoandro.

One female larva 753a (on slide) and 16 larvae (P0753), Madagascar Bas. Antongombato, Loc. small dyke near Camp Roussette (Montagne d'Ambre), Long. 49 $10^{\prime} 21^{\prime \prime} \mathrm{E}$, Lat. 12०31'27" S, Alt. $1100 \mathrm{~m}, 18.3 .1999$. J.-L. Gattolliat and Z. Rabeantoandro.

2 larvae (P0754), Madagascar, Bas. Antongombato, Riv. Makis, Loc. Waterfall of the Antakarana (Montagne d'Ambre), Long. $49^{\circ} 10^{\prime} 20^{\prime \prime} \mathrm{E}$, Lat. $12^{\circ} 31^{\prime} 09^{\prime \prime} \mathrm{S}$, Alt. $1000 \mathrm{~m}$, 18.3.1999. J.-L. Gattolliat and Z. Rabeantoandro.

3 larvae (P0829), same locality as P0754, 26.3.1999. J.-L. Gattolliat and Z. Rabeantoandro.

One female larva 757a and 5 larvae, Madagascar, Bas. Antongombato, tributary riv. of Makis, Loc. road JoffrevilleCamp Roussette (Montagne d'Ambre), Long. 49 $10^{\prime} 47^{\prime \prime} \mathrm{E}$, Lat. 12 $30^{\circ} 54^{\prime \prime}$ S, Alt. 990 m, 19.3.1999. J.-L. Gattolliat and Z. Rabeantoandro.

One larva (P811), Madagascar, Antongombato Bas., Riv. Makis, Loc. Camp Roussette (Montagne d'Ambre), Long. $49^{\circ} 10^{\prime} 21^{\prime \prime}$ E, Lat. 12³1'38" S, Alt. 1050 m, 21.3.1999. J.-L. Gattolliat and Z. Rabeantoandro.

\section{Etymology}

The specific epithet is Latin expressing the region from which the type-specimens where collected (Montagne d'Ambre).

\subsection{Labiobaetis nigrocercus sp. $n$.}

\section{Nymph}

Maximal length, fully grown female: Body $7.2 \mathrm{~mm}$. Cerci $5.0 \mathrm{~mm}$. Terminal filament $2.5 \mathrm{~mm}$. Fully grown male: Body $6.5 \mathrm{~mm}$. Cerci and terminal filament broken.

Head. Coloration pale yellow without vermiform marking on vertex and frons. Antennae pale. Turbinate eyes light brown.

Mouthparts similar to figures 15 to 20 , except labial palp with a broad thumb-like distomedial projection laterally straight (Fig. 29).

Thorax. Coloration uniformly pale yellow.

Hindwing pads present.

Legs uniformly pale yellow.

Forelegs (Fig. 30), coxa with a single stout seta on distal margin.

Femora with dorsally a row of about 25 pointed setae, regularly distributed with-few patches of 2 to 4 small and thin setae; apicodorsal patch formed by a single seta similar to those of dorsal margin; apex with about 15 short setae, some of them acute and others spatulated; ventral margin with numerous acute setae.

Tibiae and tarsi similar to figure 24 .

Abdomen. Coloration pale yellowish brown, without pattern.

Terga similar to figure 22 .

Gills present on abdominal segments 1 to 7 , tracheation dark brown well-developed, margin brown except distally, serrated distally, first gill reduced to strongly reduced, generally without tracheation.

Paraproct similar to figure 23.

Cerci yellow, except median segments brown giving a banded appearance.

\section{Adult}

Unknown

\section{Material examined}

Holotype

Male larva (P0752), Madagascar, Bas. Antongombato, Riv. Makis, Loc. Camp Roussette, Sacred Waterfall (Montagne d'Ambre), Long. $49^{\circ} 10^{\prime} 09^{\prime \prime} \mathrm{E}$, Lat. $12^{\circ} 31^{\prime} 40^{\prime \prime} \mathrm{S}$, Alt. $1075 \mathrm{~m}, 17.3 .1999$. J.-L. Gattolliat and Z. Rabeantoandro.

\section{Paratypes}

One larva 752a (on slide), same data as holotype.

One female larva $818 \mathrm{c}$ (on slide) and 2 larvae, same locality as holotype, 13.3.1999. J.-L. Gattolliat and Z. Rabeantoandro.

One larva (P0819), same locality as holotype, 13.3.1999. J.-L. Gattolliat and Z. Rabeantoandro.

6 larvae (P0825), same locality as holotype, 25.3.1999. J.L. Gattolliat and Z. Rabeantoandro.

3 larvae (P0826), same locality as holotype, 25.3.1999. J.L. Gattolliat and Z. Rabeantoandro.

One larva (P0753), Madagascar Bas. Antongombato, Loc. small dyke near Camp Roussette (Montagne d'Ambre), Long. " $49^{\circ} 10^{\prime} 21^{\prime \prime}$ E, Lat. $12^{\circ} 31^{\prime} 27^{\prime \prime}$ S, Alt. $1100 \mathrm{~m}$, 18.3.1999. J.-L. Gattolliat and Z. Rabeantoandro.

One larva (P0757), Madagascar, Bas. Antongombato, tributary riv. of Makis, Loc. road Joffreville-Camp Roussette (Montagne d'Ambre), Long. $49^{\circ} 10^{\prime} 47^{\prime \prime} \mathrm{E}$, Lat. $12^{\circ} 30^{\prime} 54^{\prime \prime} \mathrm{S}$, Alt. $990 \mathrm{~m}, 19.3 .1999$. J.-L. Gattolliat and Z. Rabeantoandro.

16 larvae (P0812), Madagascar, Bas. Antongombato, Riv. Makis, Loc. Great Waterfall (Montagne d'Ambre), Long. $49^{\circ} 10^{\prime} 14^{\prime \prime}$ E, Lat. 12²9'23" S, Alt. 690 m, 22.3.1999. J.-L. Gattolliat and Z. Rabeantoandro.

One female larva 814b and 9 larvae (P0814), Madagascar, Bas. Antongombato, Riv. Makis, Loc. 100m down stream the Great Waterfall (Montagne d'Ambre), Long. 49 $10^{\prime} 14^{\prime \prime}$ E, Lat. $12^{\circ} 29^{\prime} 17^{\prime \prime}$ S, Alt. $675 \mathrm{~m}, 22.3 .1999$. J.-L. Gattolliat and Z. Rabeantoandro. 


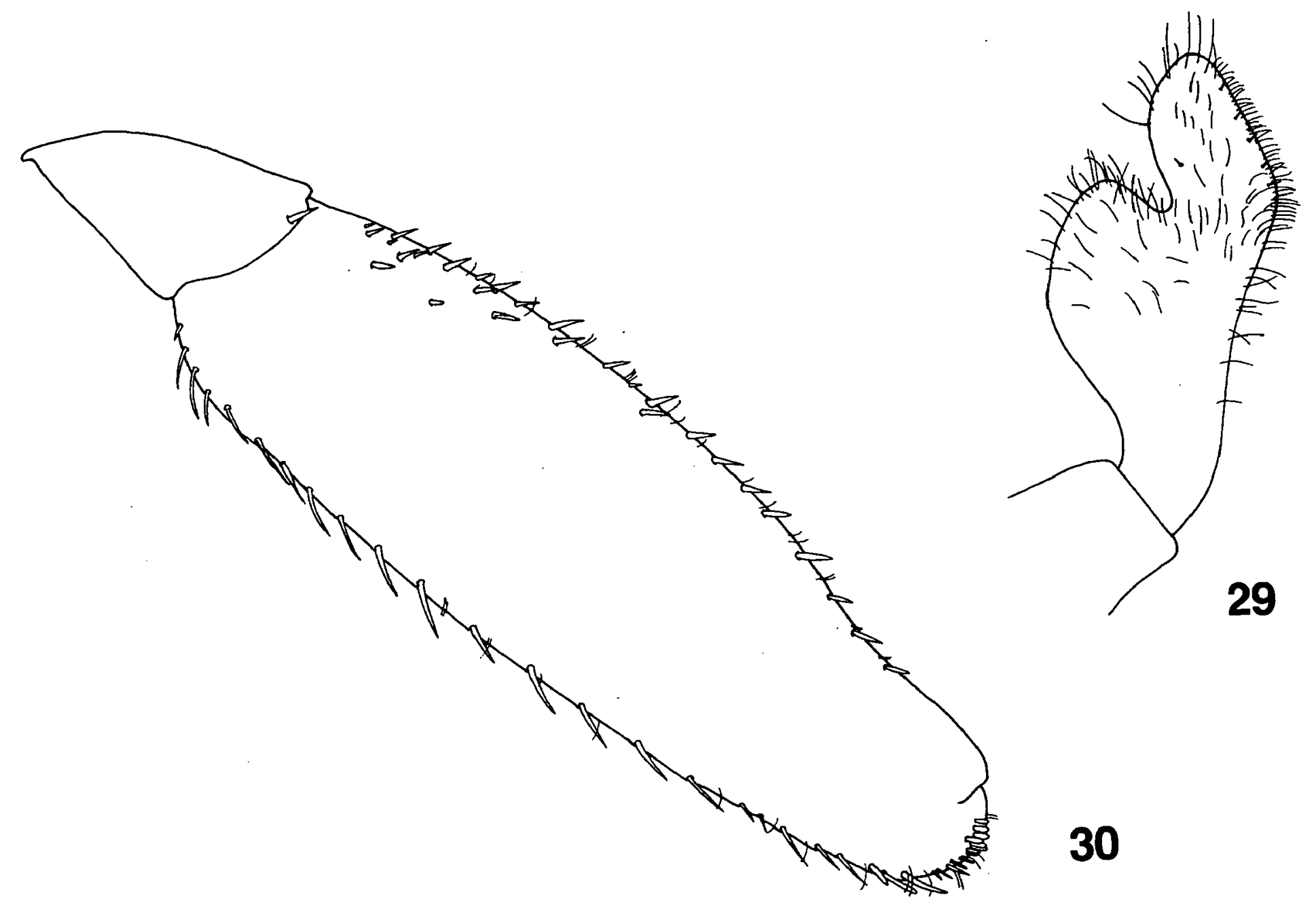

Figs. 29 to 30. Larval structures of $L$. nigrocercus sp. n. : 29 : second and third segments of labial palp. 30 : forefemur.

Figs. 29 a 30. Structures larvaires de L. nigrocercus n. sp. : 29 : second et troisième segments du palpe labial. 30 : fémur antérieur.

8 larvae (P0822), same locality as P0814, 24.3.1999. J.-L. Gattolliat and Z. Rabeantoandro.

\section{Other material}

One male larva 675a (on slide) and one larva (P0675), Madagascar, Bas. Betsiboka, Riv. Sahavilana, Long. 47 $57^{\prime 2} 20^{\prime \prime}$ E, Lat. $18^{\circ} 28^{\prime} 47^{\prime \prime}$ S, Alt. 1375 m,14.12.1999. D. Randriamasimanana.

\section{Etymology}

The specific epithet is Latin expressing the broad and dark stripe on the cerci.

\subsection{Labiobaetis vulgaris sp. $n$.}

\section{Nymph}

Size variable between different populations. Maximal length, fully grown female: Body $4.9 \mathrm{~mm}$. Cerci $3.4 \mathrm{~mm}$. Terminal filament $2.0 \mathrm{~mm}$. Fully grown male: Body $4.5 \mathrm{~mm}$. Cerci $3.4 \mathrm{~mm}$. Terminal filament 2.0 $\mathrm{mm}$.
Head. Coloration pale yellowish brown, with vermiform marking on vertex and frons, border of sclerites yellow. Antennae pale yellow; pedicellus without distolateral process. Turbinate eyes light brown.

Labrum (Fig. 31) rounded and narrow, with a wellmarked anteromedial emargination, dorsally with an arc of about 10 feathered setae, long and thin setae abundant on whole dorsal face; distal margin bordered with feathered setae; ventrally with 3 stout, minute setae and a disto-medial arc of very thin setae.

Right mandible (Fig. 32), stout prostheca apically with rounded denticules; margin between prostheca and mola crenate, with a well-developed hump, without setae; tuft of setae at apex of mola reduced to a small setae; basal half with dorsally long and thin setae.

Left mandible (Fig. 33), stout prostheca apically with denticules and a well-developed comb-shape structure; margin between prostheca and mola crenate, slightly concave without hump and without setae; tuft 


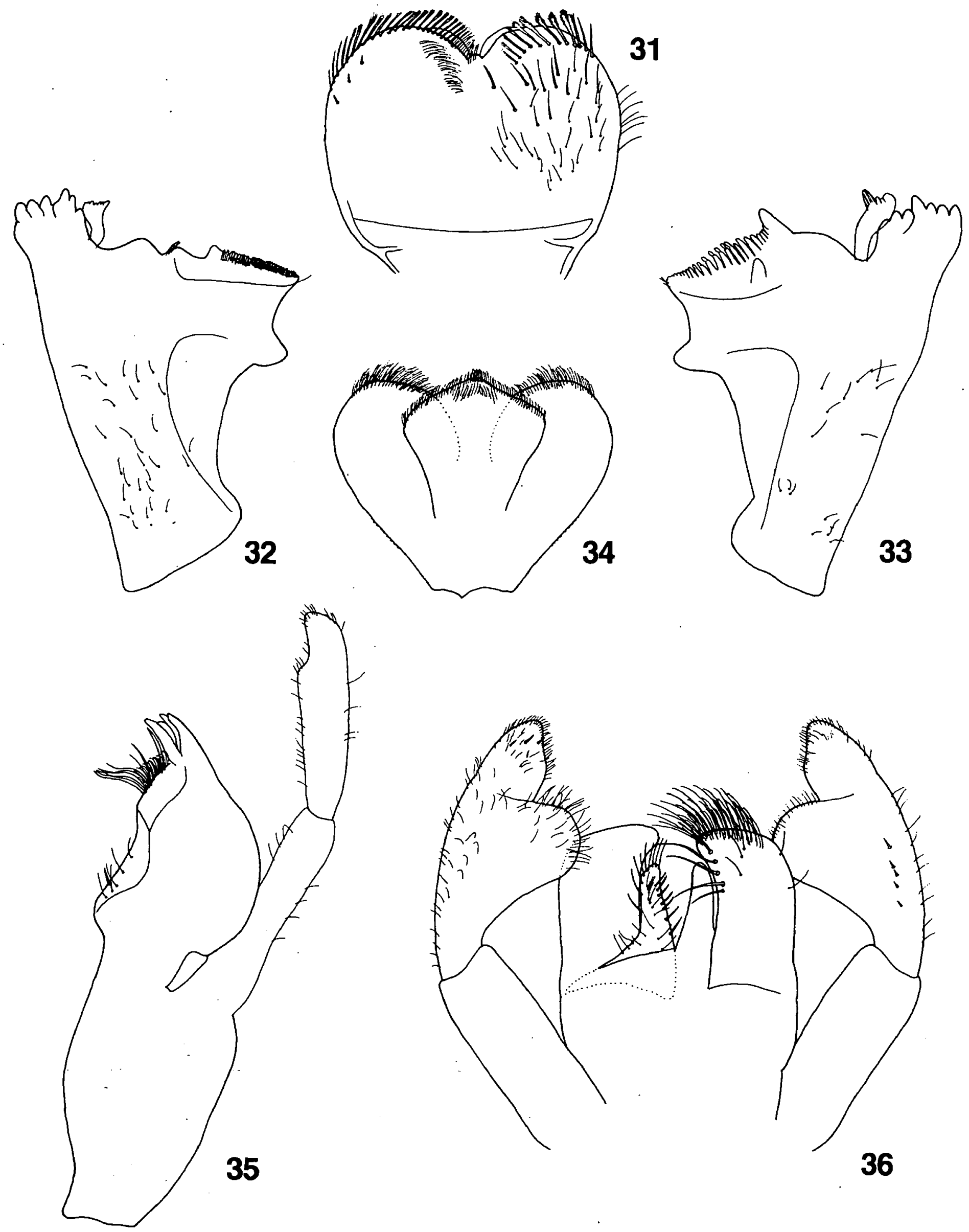

Figs. 31 to 36 . Larval structures of $L$. vulgaris sp. n. : 31 : labrum (left : ventral; right : dorsal). $32:$ right mandible. $33:$ left mandible. 34 : hypopharynx. 35 : left maxilla. 36 : labium.

Figs. 31 à 36 . Structures larvaires de $L$. vulgäris n. sp. : 31 : labre (gauche : ventral; droite : dorsal). 32 : mandibule droite. 33 : mandibule gauche. 34 : hypopharynx. 35 : maxille gauche. 36 : labium. 
of setae at apex of mola reduced to few setae; basal half with dorsally few long and thin setae.

Hypopharynx (Fig. 34), superlingua triangular without a distinct median projection, covered with thin setae and few black stout ones medially.

Maxillae (Fig. 35) with 4 subequal teeth, none of them opposed to others; row of setae ending with 6 long setae, 2 spine-like setae in middle of row; row of 4 small setae at basis of galea; one single seta perpendicular to margin of galea; palp 2-segmented, longer than galealacinia, segment 2 with a weakly pronounced distomedial concavity.

Labium (Fig. 36) with glossae clearly shorter than paraglossae; glossae stout, lateral margins with setae longer apically, stouter on inner margin, row of 7 setae medially; paraglossae stout, almost rectangular, apically flattened, with 3 to 4 rows of pectinate setae, inner margin with 5 very long setae. Labial palp 3-segmented; first segment slender, subrectangular, $0.75 \mathrm{x}$ length of second and third combined; second segment with a well-developed thumb-like distomedial projection, covered with thin setae, row of 5 short and stout setae dorsally; third segment subconical, inner margin slightly concave apically, outer margin convex, ventral surface covered with short thin setae, dorsal surface with short fine setae apically.

Thorax. Prothorax pale yellowish, some specimens among same population darker.

Hindwing pads absent.

Legs uniformly pale yellow.

Forelegs (Fig. 40), coxa with 3 to 4 stout setae on ventral margin.

Femora with dorsally a row of about 10 pointed setae, rare and long in distal part, smaller and more abundant proximally; apicodorsal patch formed by 2 long setae; apex with 5 to 8 short spatulated setae; ventral margin with blunt setae, rare apically.

Tibiae dorsally with minute spatulated setae; apically with a single stout blunt seta; ventrally with pointed setae, very abundant apically; tibio-patellar suture present.

Tarsi with only few thin setae and few minute ones dorsally; ventral margin with a row of pointed setae increasing in length toward apex; tarsal claws hooked (Fig. 41) with one row of about 11 small teeth, longer ones in middle; subapical setae absent.

Second and third legs similar to foreleg.

Abdomen. Terga pale brown, lighter medially; darker specimens with 4 yellow small spots on each tergum; proximal margin bordered by a black line inter- rupted medially; terga not shagreen with scale bases and setae (Fig. 38); posterior margin with pointed, triangular spination broader than long.

Sterna pale yellow, with few scale bases and setae; smooth posterior margin without spines, except sterna 7 to 9 with triangular irregular spination.

Gills (Fig. 37) present on abdominal segments 2 to 7 , tracheation brown poorly developed, serrated distally.

Paraproct (Fig. 39) with about 75 small scale bases and few setae, margin with 20 irregular spines; postero-lateral extension with about 6 scale bases, 20 blunt spines along margin.

Cerci yellow, except median segments brown giving a banded appearance.

\section{Adult}

Maximal length, female imago: Body $4.7 \mathrm{~mm}$. Forewing $4.9 \mathrm{~mm}$. Male imago: Body $4.3 \mathrm{~mm}$. Forewing $4.5 \mathrm{~mm}$.

Head. Yellowish brown. Turbinate eyes uniformly honey brown. Eyes black. Antennae pale yellow.

Thorax. Copper brown.

Forewings $3.9 \mathrm{~mm}$, hyaline; double intercalary veins between longitudinal veins; pterostigma with 4 to 6 cross-veins generally not reaching subcostal vein (Fig. 42).

Hindwings absent.

Legs uniformly pale yellow.

Terga brown lighter medially. .

Sterna pale yellow.

Male genitalia (Fig. 43) with three-segmented gonopods, first and second segments almost fused, first segment without apophysis, third segment globular; small sclerotized knob-shaped process between forceps.

\section{Material examined}

\section{Holotype}

Female larva (P0836), Madagascar, Bas. Antongombato, Riv. Antongombato, Loc. Namakia, Long. 49 $14^{\prime} 37^{\prime \prime}$ E, Lat. $12^{\circ} 19^{\prime} 32^{\prime \prime}$ S, Alt. $20 \mathrm{~m}, 30.3 .1999$. J.-L. Gattolliat and Z. Rabeantoandro.

\section{Paratypes}

One female larva 836a (on slide) and seventeen larvae (P0836), same data as holotype.

One female larva 834a (on slide) and fifteen larvae (P0834), Madagascar, Bas. Antongombato, Loc. between Diégo-Suarez and Namakia, Long. $49^{\circ} 16^{\prime} 50^{\prime \prime} \mathrm{E}$, Lat. $12^{\circ} 19^{\prime} 40^{\prime \prime}$ S, Alt. $40 \mathrm{~m}, 30.3 .1999$. J.-L. Gattolliat and Z. Rabeantoandro. 

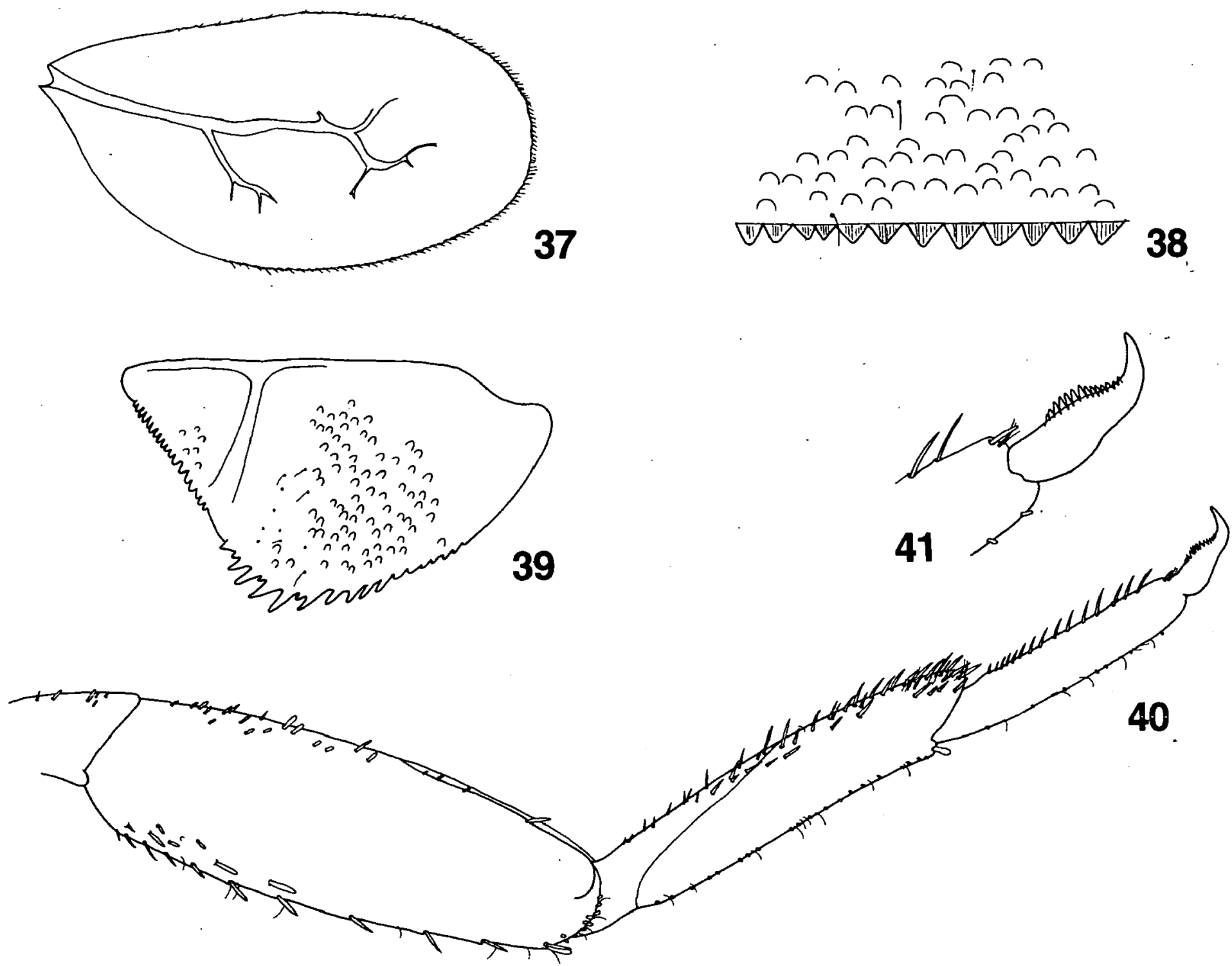

Figs. 37 to 41 . Larval structures of $L$. vulgaris sp. n. : 37 : fourth gill. 38 : detail of tergum. 39 : paraproct. $40:$ left foreleg. 41 : tarsal claw.

Figs. 37 à 41 . Structures larvaires de $L$. vulgaris n. sp. : 37 : quatrième branchie. 38 : détail d'un tergite. 39 : paraprocte. 40 : première patte gauche. 41 : griffe tarsale.

One female larva 221a (on slide) and two larvae (P0221), Madagascar, Bas. Namorona, tributary of Namorona, Loc. 1 $\mathrm{km}$ from Vohiparara, Long. $47^{\circ} 22^{\prime} 43^{\prime \prime} \mathrm{E}$, Lat. $21^{\circ} 13^{\prime} 53^{\prime \prime} \mathrm{S}$, Alt. 1200 m, 20.4.1994. J.-M. Elouard.

One female larva 381a (on slide) and 17 larvae (P0381), Madagascar, Bas. Mangoro, Riv. Mandraka tributary of Mangoro, Loc. $2 \mathrm{~km}$ from Ambodinifody, Long. $48^{\circ} 03^{\prime} 12^{\prime \prime}$ E, Lat. 18 $8^{\circ} 2^{\prime} 06^{\prime \prime}$ S, Alt. $1060 \mathrm{~m}, 29.4 .1995$. L. Ruffieux and M. Sartori.

One male larva 432a (on slide) and nine larvae (P0432), Madagascar, Bas. Mangoky, Riv. Malio, Loc. Beroroha, Long. $45^{\circ} 07^{\prime} 32^{\prime \prime}$ E, Lat. $21^{\circ} 40^{\prime} 31^{\prime \prime}$ S, Alt. 155 m, 29.5.1995. J.-M. Elouard and R. Oliarinony.

One female larva 439a (on slide) and six larvae (P0439), Madagascar, Bas. Onilahy, Riv. tributary of Thazofotsy, Loc.
Ambatofotsy (Horombe), Long. $45^{\circ} 40^{\prime} 43^{\prime \prime}$ E, Lat. $22^{\circ} 30^{\prime} 49^{\prime \prime}$ S, Alt. 910 m, 1.6.1995. J.-M. Elouard.

One male imago 441a (on slide), nine male imagoes and thirteen female imagoes (P0441), Madagascar, Bas. Manampatrana, Riv. Namorana tributary of Manampatrana, Loc. Tsararano, Long. $47^{\circ} 20^{\prime} 11^{\prime \prime}$ E, Lat. $22^{\circ} 48^{\prime} 55^{\prime \prime}$ S, Alt. $100 \mathrm{~m}$, 15.6.1995. M.R. Andriamihaja and A. Ralaiteferana.

Twenty two larvae (P0442), Madagascar, Bas. Manampatrana, Riv. Namorana, Loc. Tsararano, Long. $47^{\circ} 20^{\prime} 43^{\prime \prime}$ E, Lat. $22^{\circ} 48$ '23" S, Alt. 100 m, 15.6.1995. M.R. Andriamihaja and A. Ralaiteferana.

One female larva 460a (on slide) and eleven larvae -(P0460), Madagascar, Bas. Matitanana, Riv. Ariambola Aff. de Manambondro, Loc. bridge to Ariambola, Long. 


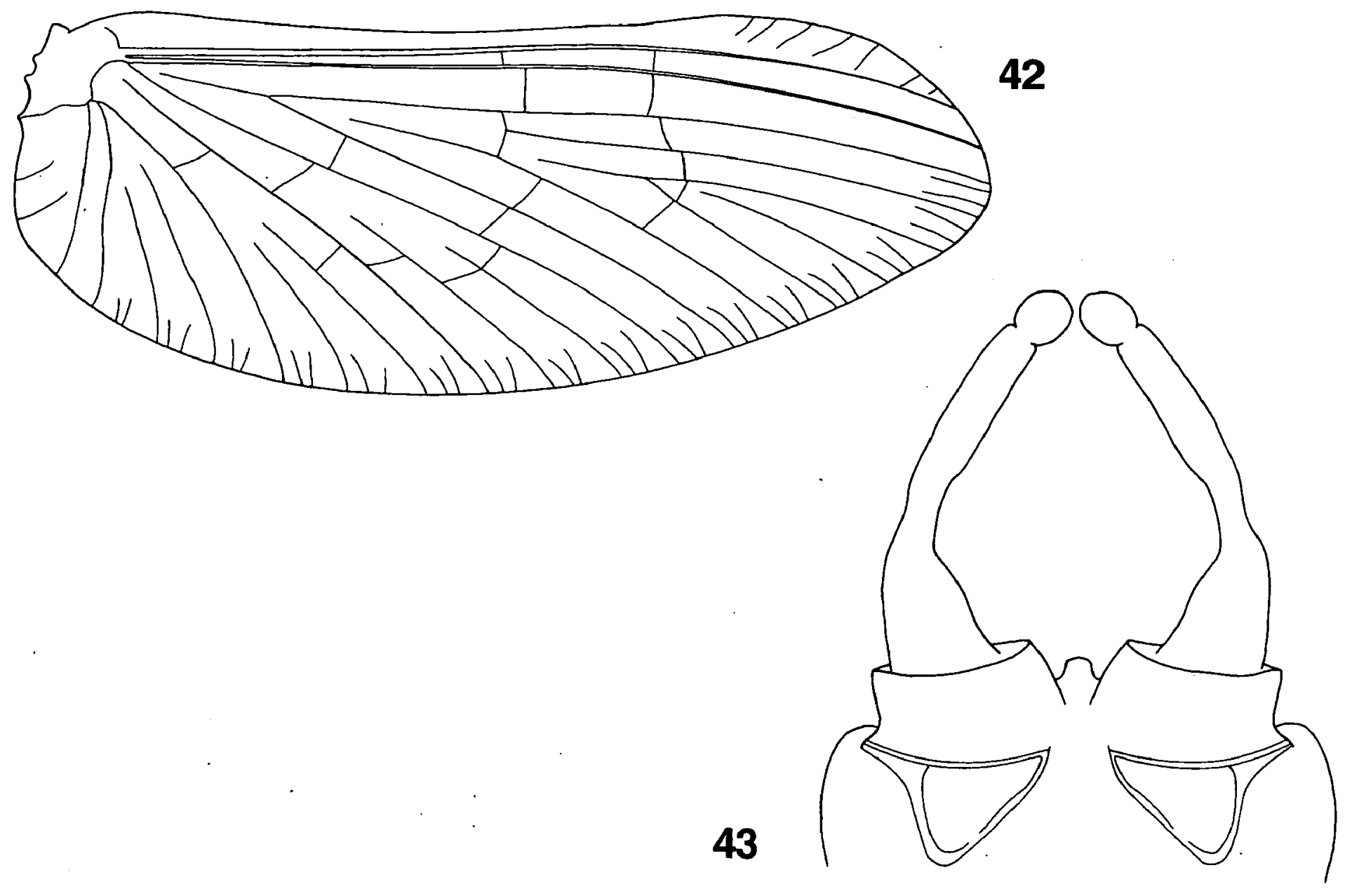

Figs. 42 to 43 . Imaginal male structures of $L$. vulgaris sp. n. : $42:$ forewing. 43 : genitalia.

Figs. 42 à 43 . Structures imaginales males de $L$. vulgaris $n$. sp. : 42 : aile antérieure. 43 : génitalia.

$47^{\circ} 28^{\prime} 46^{\prime \prime}$ E, Lat. 22¹1'04" S, Alt. 30 m, 22.6.1995. M.R. Andriamihaja and A. Ralaiteferana.

One male larva 470a (on slide) and two female larvae (P0470), Madagascar, Bas. Tsiribihina, Riv. Amananara, Loc. Andranomiady, Long. 46 59'15" E, Lat. $19^{\circ} 14^{\prime} 40^{\prime \prime} \mathrm{S}$, Alt. 1270 m, 5.10.1995. M.R. Andriamihaja and A. Ralaiteferana.

Two female larvae 472a and 472b (on slide) and one hundred larvae (P0472), Madagascar, Bas. Tsiribihina, Riv. Kitsamby tributary of Tsiribihina, Loc. Andakana, Long. 46 ${ }^{\circ} 46^{\prime} 23^{\prime \prime}$ E, Lat. 19¹5'33" S, Alt. 1130 m, 7.10.1995. M.R. Andriamihaja and A. Ralaiteferana.

One male larva 488a (on slide) and eight larvae (P0488), Madagascar, Bas. Betsiboka, Riv. Amberomanga, Loc. Amboasary, Long. $47^{\circ} 56^{\prime} 17^{\prime \prime}$ E, Lat. $18^{\circ} 28^{\prime} 03^{\prime \prime}$ S, Alt. $1270 \mathrm{~m}$, 19.10.1995. F.M. Gibon.

One male larva 650a (on slide) and seven larvae (P0650), Madagascar, Bas. Mananjary, Riv. Vintanona, Loc. Andranomaitso, Long. $47^{\circ} 45^{\prime} 14^{\prime \prime}$ E, Lat. $21^{\circ} 20^{\prime} 37^{\prime \prime} \mathrm{S}$, Alt. $320 \mathrm{~m}$, 12.11.1996. J.-L. Gattolliat.

One male imago 661a (on slide) and two male imagoes (P0661), Madagascar, Bas. Betsiboka, Riv. Mananara,

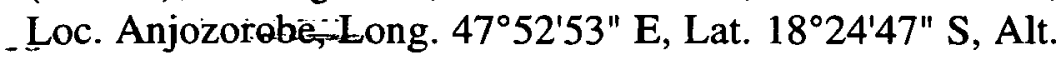
$1220 \mathrm{~m}, 16.11 .1996 . \mathrm{R}$. Oliarinony.
One male larva 869a (on slide) and one female larva 869b (on slide), Madagascar, Bas. Mangoro, Riv. Mangoro, Loc.Mangoro (bridge). Long. $48^{\circ} 06^{\prime} 32^{\prime \prime} \mathrm{E}$, Lat. $18^{\circ} 52^{\prime} 32^{\prime \prime} \mathrm{S}$, Alt. $840 \mathrm{~m}, 12.4 .1999$. J.-L. Gattolliat and N. Raberiaka.

\section{Other material}

One larva (P0004), Madagascar, Bas. Rianila, Riv. Aff. Sahatandra, Loc. Moramanga, Long. 48 ${ }^{\circ} 9^{\prime} 12^{\prime \prime}$ E, Lat. $18^{\circ} 56^{\prime} 27^{\prime \prime}$ S, Alt. 800 m, 13.7.1990. J.-M. Elouard.

One female larva 28a (on slide) and twenty two larvae (P0028), Madagascar, Bas. Onilahy, Riv. Imaloto, Long. $45^{\circ} 23^{\prime} 43^{\prime \prime}$ E, Lat. $22^{\circ} 35^{\prime} 45^{\prime \prime}$ S, Alt. 780 m, 7.4.1991. J.-M. Elouard.

Three larvae (P0044), Madagascar, Bas. Betsiboka, Riv. Andranofeno Sud, Long. $47^{\circ} 10^{\prime} 46^{\prime \prime}$ E, Lat. $18^{\circ} 05^{\prime} 00^{\prime \prime} \mathrm{S}$, Alt. 1425 m, 17.4.1991. J.-M. Elouard.

Two larvae (P0101), Madagascar, Bas. Mangoky, Riv. Sahambano, Loc. Ankaramena, Long. 46 $37^{\prime} 11^{\prime \prime} \mathrm{E}$, Lat. $22^{\circ} 03^{\prime} 28^{\prime \prime}$ S, Alt. 775 m, 21.4.1992. J.-M. Elouard.

One larva (P0239), Madagascar, Bas. Mangoky, tributary

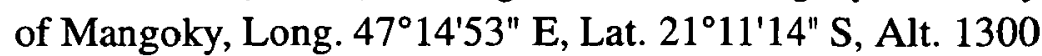
$\mathrm{m}$, 27.4.1994. J.-M. Elouard. 
One larva (P0302), Madagascar, Bas. Mangoky, Riv. Malio, Loc. Bereketa, near Ranohira, Long. $45^{\circ} 08^{\prime} 28^{\prime \prime} \mathrm{E}$, Lat. $22^{\circ} 32^{\prime} 02^{\prime \prime}$ S, Alt. 645 m, 19.4.1994. J.-M. Elouard.

One larva (P0307), Madagascar, Bas. Mangoky, Riv. Menamaty, Loc. Ranohira, Long. 45 $24^{\prime} 07^{\prime \prime} \mathrm{E}$, Lat. $22^{\circ} 33^{\prime} 03^{\prime \prime} \mathrm{S}$, Alt. 760 m, 22.4.1994. J.-M. Elouard.

One larva (P0380), Madagascar, Bas. Mangoro, tributary of Mangoro, Loc. $1 \mathrm{~km}$ from Sahafitahana, Long. 48 $13^{\prime} 39^{\prime \prime}$ E, Lat. $18^{\circ} 59^{\prime} 42^{\prime \prime}$ S, Alt. 880 m, 26.4.1995. L: Ruffieux and M. Sartori

Three larvae (P0384), Madagascar, Bas. Tsiribihina, Riv. Ampasina tributary of Mania, Loc. near Mahatsanda, Long. $47^{\circ} 06^{\prime} 54^{\prime \prime}$ E, Lat. $20^{\circ} 15^{\prime} 57^{\prime \prime}$ S, Alt. 1380 m, 15.5.1995. F.M. Gibon.

One female larva 427a (on slide) and five larvae (P0427), Madagascar, Bas. Onilahy, Riv. Sahavatoy tributary of Ilanana, Loc. Ampandrabe, Long. 45 43'45" E, Lat. $22^{\circ} 38^{\prime} 24^{\prime \prime} \mathrm{S}$, Alt. 910 m, 26.5.1995. J.-M. Elouard and R. Oliarinony.

Fourteen larvae (P0434), Madagascar, Bas. Mangoky, Riv. Isahahena, Loc. Berenty-Betsileo, Long. $45^{\circ} 03^{\prime} 59^{\prime \prime} \mathrm{E}$, Lat. $22^{\circ} 09^{\prime} 52^{\prime \prime}$ S, Alt. 385 m, 30.5.1995. J.-M. Elouard and R. Oliarinony.

Twelve larvae (P0445), Madagascar, Bas. Manampatrana, Riv. Manandavana, Loc. Mahatsinjo-Be (Bac), Long. $47^{\circ} 21^{\prime} 13^{\prime \prime}$ E, Lat. $22^{\circ} 28^{\prime} 57^{\prime \prime}$ S, Alt. 210 m, 17.6.1995. M.R. Andriamihaja and A. Ralaiteferana.

One larva (P0466), Madagascar, Bas. Betsiboka, Riv. Onibe (1er bras), Loc. Arivonimamo, Long. $47^{\circ} 08^{\prime} 15^{\prime \prime} \mathrm{E}$, Lat. $18^{\circ} 58^{\prime} 40^{\prime \prime}$ S, Alt. 1270 m, 3.10.1995. M.R. Andriamihaja and A. Ralaiteferana.

Three larvae (P0469), Madagascar, Bas. Tsiribihina, Riv. Kitsamby, Loc. Morarano nord, Long. $47^{\circ} 00^{\prime} 00^{\prime \prime} \mathrm{E}$, Lat. $19^{\circ} 16^{\prime} 50^{\prime \prime}$ S, Alt. 1200 m, 5.10.1995. M.R. Andriamihaja and A. Ralaiteferana.

Two larvae (P0473), Madagascar, Bas. Tsiribihina, Riv. Lily tributary of Mahajilo, Loc. Antafofo, Long. $46^{\circ} 41^{\prime} 07^{\prime \prime}$ E, Lat. $19^{\circ} 01^{\prime} 43^{\prime \prime}$ S, Alt. 1100 m, 8.10.1995. M.R. Andriamihaja and A. Ralaiteferana.

Three larvae (P0477), Madagascar, Bas. Tsiribihina, Riv. Mazy tributary of Sakay, Loc. Amparaky RN1, Long. 46³8'07" E, Lat. $18^{\circ} 56^{\prime} 03^{\prime \prime}$ S, Alt. 975 m, 11.10.1995. M.R. Andriamihaja and A. Ralaiteferana.

Twelve larvae (P0581), Madagascar, Bas. Tsiribihina, Loc. Fenomanana, Long. $47^{\circ} 01^{\prime} 17^{\prime \prime} \mathrm{E}$, Lat. $19^{\circ} 30^{\prime} 05^{\prime \prime} \mathrm{S}$, 11.4.1996. J.-M. Elouard and R. Oliarinony.

Five larvae (P0582), Madagascar, Bas. Tsiribihina, Loc. Amparihy, Long. 4659'53" E, Lat. $19^{\circ} 27^{\prime} 07^{\prime \prime}$ S, Alt. 1900 m, 12.4.1996. J.-M. Elouard and R. Oliarinony.

Five larvae (P0594), Madagascar, Bas. Tsiribihina, Long. $46^{\circ} 01^{\prime} 50^{\prime \prime}$ E, Lat. $19^{\circ} 36^{\prime} 10^{\prime \prime}$ S, Alt. 820 m, 19.5.1996. F.M. Gibon.

Three larvae (P0595), Madagascar, Bas. Tsiribihina, Loc. Ilaka-Afovoany, Long. $47^{\circ} 10^{\prime} 35^{\prime \prime} \mathrm{E}$, Lat. $20^{\circ} 22^{\prime} 00^{\prime \prime} \mathrm{S}$, Alt. 1455 m, 21.5.1996. J.-M. Elouard.
One larva (P0602), Madagascar, Bas. Maharivo, Riv. Maharivo, Loc. Befasy, Long. $44^{\circ} 20^{\prime} 45^{\prime \prime} \mathrm{E}$, Lat. $20^{\circ} 34^{\prime} 18^{\prime \prime} \mathrm{S}$, Alt. 50 m, 27.5.1996. J.-M. Elouard.

One larva (P0636), Madagascar, Bas. Namorona, Riv. Tsaratango, Loc. Tsaratango, Long. $47^{\circ} 31^{\prime} 50^{\prime \prime} \mathrm{E}$, Lat. $21^{\circ} 16^{\prime} 33^{\prime \prime}$ S, Alt. 585 m, 8.11.1996. J.-L. Gattolliat and C. Rochat.

Two larvae and five male imagoes (P0651), Madagascar, Bas. Manjary, Loc. Andranomaitso, Long. $47^{\circ} 45^{\prime} 07^{\prime \prime}$ E, Lat. $21^{\circ} 20^{\prime} 40^{\prime \prime}$ S, Alt. $320 \mathrm{~m}, 12.11 .1996$. J.-L. Gattolliat.

Two larvae (P0718), Madagascar, Bas. Mangoro, Riv. Mangoro, Loc. Mangoro (bridge), Long. 48 06'32" E, Lat. 18 52'32" S, Alt. 840 m, 28.4.1998. J.-M. Elouard and M. Sartori.

Two larvae (P0726), Madagascar, Bas. Rianila, Riv. Sandrakatrana, Loc. Ambodiaviavy, Long. 48 $38^{\prime} 50^{\prime \prime}$ E, Lat. $18^{\circ} 57^{\prime} 50^{\prime \prime}$ S, Alt. 420 m, 30.4.1998. M. Sartori.

P0812, Madagascar, Bas. Antongombato, Riv. Makis, Loc. Great Fall (Montagne d'Ambre). Long. 49 $10^{\prime} 14^{\prime \prime} \mathrm{E}$, Lat. $12^{\circ} 29^{\prime} 23^{\prime \prime}$ S, Alt.690 m, 22.3.1999. J.-L. Gattolliat and Z. Rabeantoandro.

One larva (P0862), Madagascar, Bas. Rianila, tributary riv. to Sahatandra Riv., Loc.near Ambalafotsy, road to Lakato. Long. $48^{\circ} 21^{\prime} 51^{\prime \prime} \mathrm{E}$, Lat. $19^{\circ} 02^{\prime} 22^{\prime \prime} \mathrm{S}$, Alt.1050 m, 8.4.1999. J.-L. Gattolliat and N. Raberiaka.

Seventeen larvae (P0863), Madagascar, Bas. Rianila, Riv. Sandrakatrana, Loc. Ambodiaviavy, near Beforona. Long. $48^{\circ} 39^{\prime} 45^{\prime \prime}$ E, Lat. $18^{\circ} 57^{\prime} 31^{\prime \prime}$ S, Alt.350 m, 9.4.1999. J.-L. Gattolliat and N. Raberiaka.

\section{Etymology}

The specific epithet is Latin expressing the abundance and the wide distribution of the species.

\subsection{Labiobaetis punctatus sp. $\mathrm{n}$.}

\section{Nymph}

Maximal length, fully grown female: Body $5.2 \mathrm{~mm}$. Cerci $3.8 \mathrm{~mm}$. Terminal filament $2.1 \mathrm{~mm}$. Fully grown male: Body $4.4 \mathrm{~mm}$. Cerci $3.4 \mathrm{~mm}$. Terminal filament $1.8 \mathrm{~mm}$.

Head. Coloration almost uniformly pale brown, vermiform marking on vertex and frons. Antennae light yellow; pedicellus without distolateral process. Turbinate eyes light brown.

Labrum (Fig. 44) rounded, with an anteromedial emargination, dorsally with an arc of about 17 stout setae, long and thin setae medially; distal margin bordered with setae, disto-lateral ones feathered; ventrally with 4 stout, minute setae and a disto-medial arc of very thin setae.

Right mandible (Fig. 45), stout prostheca apically with rounded denticules; margin between prostheca and mola poorly crenate, without setae, slightly 


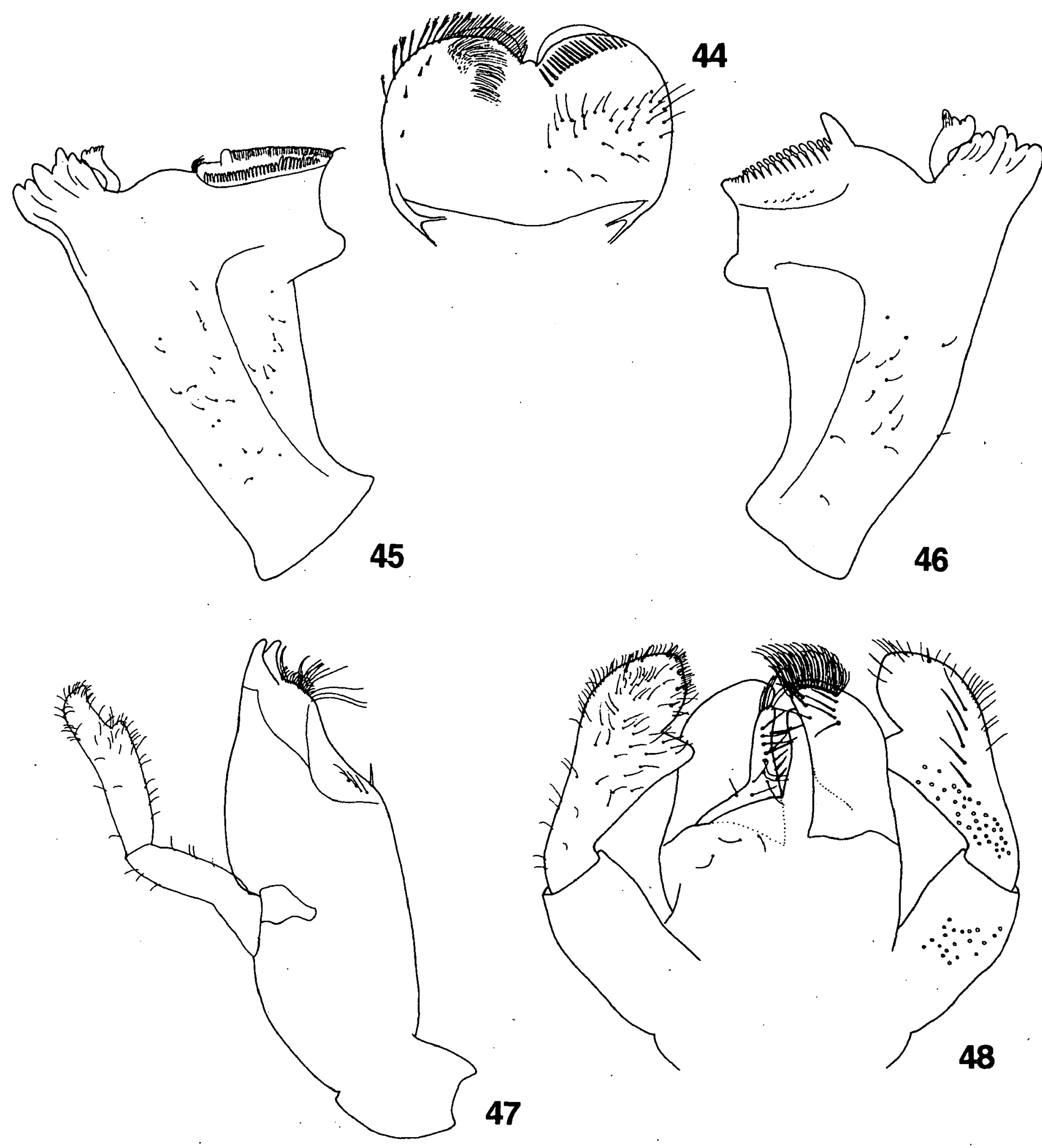

Figs. 44 to 48 . Larval structures of $L$. punctatus sp. n. : 44 : labrum (left : ventral; right : dorsal). 45 : right mandible. $46:$ left mandible. 47 : right maxilla. 48 : labium.

Figs. 44 à 48 . Structures larvaires de $L$. punctatus n. sp. : 44 : labre (gauche : ventral; droite : dorsal). $45:$ mandibule droite. 46 : mandibule gauche. $47:$ maxille droite. $48:$ labium. 
convex; tuft of setae at apex of mola reduced to two small setae; basal half with dorsally short thin setae.

Left mandible (Fig. 46), stout prostheca apically with denticules and a comb-shape structure; margin between prostheca and mola poorly crenate, slightly convex without hump and without setae; tuft of setae at apex of mola absent; basal half with dorsally thin setae.

Hypopharynx as in figure 4, superlingua with a projection medially, covered apically with stouter setae.

Maxillae (Fig. 47) with 2 small and 2 large teeth, none of them opposed to others; row of setae ending with 8 long setae, 2 spine-like setae in middle of row; row of 5 small setae at basis of galea; one single small seta perpendicular to margin of galea; palp 2-segmented, segment 2 with a pronounced distomedial concavity.

Labium (Fig. 48) with glossae clearly shorter than paraglossae; inner margin of glossae with stout setae, longer at apex, row of 5 to 6 setae medially; paraglossae stout, apically rounded, with 3 to 4 rows of pectinate setae. Mentum with 3 to 6 thin setae apically. Labial palp 3-segmented; first segment almost without setae, $0.7 \mathrm{x}$ length of second and third combined; second segment with a triangular distomedial projection, covered with thin setae, row of 5 very long setae dorsally; third segment subconical, inner and outer margins rounded and almost symmetrical, covered with short thin setae and few longer and stouter ones especially near margins.

Thorax. Pale brown, with generally 2 darker longitudinal stripes in middle of mesothorax.

Hindwing pads present.

Legs uniformly light yellow.

Forelegs (Fig. 53), coxa with 3 to 4 stout setae on distal margin.

Femora with dorsally a row formed only by 6 pointed setae and few smaller ones in proximal part; apicodorsal patch formed by 2 stout and long setae; apex with 2 short and pointed setae and 3 spatulated ones; ventral margin with numerous pointed setae, slightly longer apically.

Tibiae dorsally with only few small setae; apically with two stout pointed setae; ventrally with pointed setae, quite abundant apically; tibio-patellar suture present.

Tarsi without setae dorsally; ventral margin with a row of pointed setae increasing in length toward apex; tarsal claws (Fig. 54) with one row of about 11 teeth decreasing in length toward apex; subapical setae absent.

Second and third legs similar to foreleg.
Abdomen. Coloration middle to dark brown, lighter laterally (Fig. 49), with 4 central yellow spots, terga 3 and 6 with a yellow central pattern, terga 4 and 5 and sometimes 2 with a black spot in middle.

Terga (Fig. 51) not shagreen with scale bases; posterior margin with acute spination slightly broader than long, space between spines generally almost as broad as a spine.

Sterna pale yellow, with scale bases; smooth posterior margin without spines.

Gills (Fig. 50) present on abdominal segments 1 to 7 , tracheation brown poorly developed, deeply serrated distally, gill 1 clearly reduced.

Paraproct (Fig. 52) with about 25 scale bases, margin with about 25 pointed spines; postero-lateral extension with about 15 scale bases, well-developed pointed spines along margin.

Cerci yellow, except median segments brown giving a banded appearance.

\section{Adult}

Male subimagoes only known.

Maximal length, male subimago: Body $4.1 \mathrm{~mm}$. Forewing $3.9 \mathrm{~mm}$. Hindwing $0.6 \mathrm{~mm}$.

Head. Yellowish brown. Turbinate eyes honey brown with darker basis. Eyes black.

Thorax. Copper brown.

Forewings with pterostigma with 4 to 6 cross-veins, shape and main venation similar to figure 13 .

Hindwings narrow, crescent-shaped, without costal process, longitudinal veins not visible (Fig. 55).

Legs uniformly light yellow.

Terga pale brown with 4 yellow spots on each segment.

Male genitalia with three-segmented gonopods; well-developed sclerotized process between forceps, as broad as distance between forceps, apically flattened, covered with small thin setae (Fig. 56).

\section{Material examined \\ Holotype}

Female larva (P0337), Madagascar, Bas. Mandrare, Riv. Mananara-Sud, Loc. near Amboasary, Long. 46 $26^{\prime} 34^{\prime \prime} \mathrm{E}$, Lat. 2451'03" S, Alt. 50 m, 1.6.1994. J.-M. Elouard.

\section{Paratypes}

Three male subimagoes with corresponding larval exuvia 337-11 to 337-16 (on slides), one female larva 337b (on slide) and 13 larvae (P0337), same data as holotype.

One female larva 221a (on slide) and 3 larvae (P0221), 

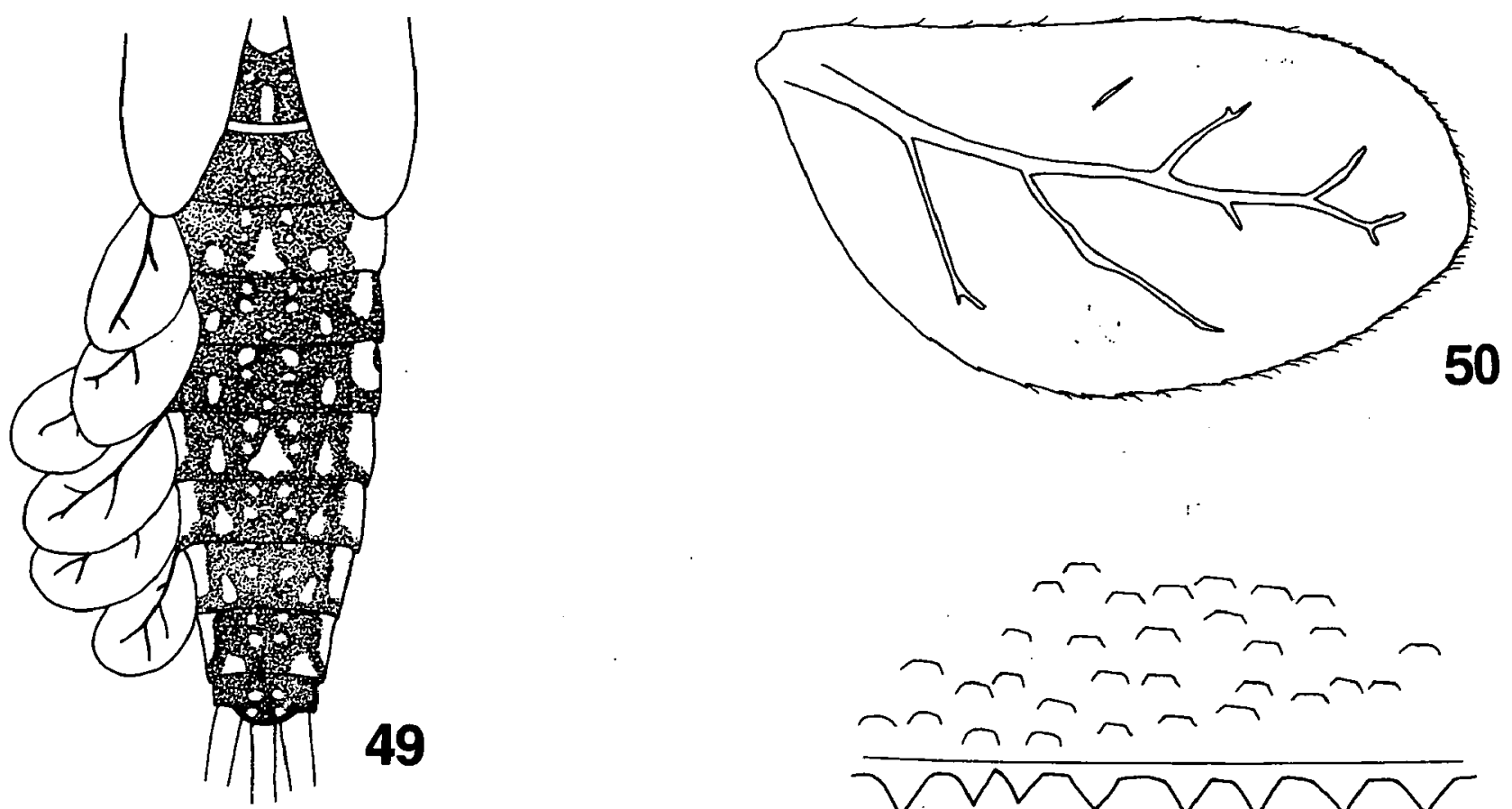

49

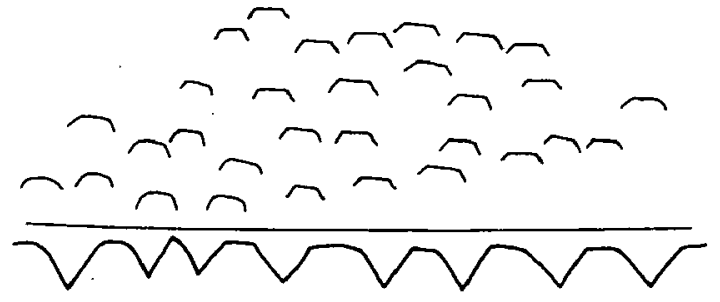

51
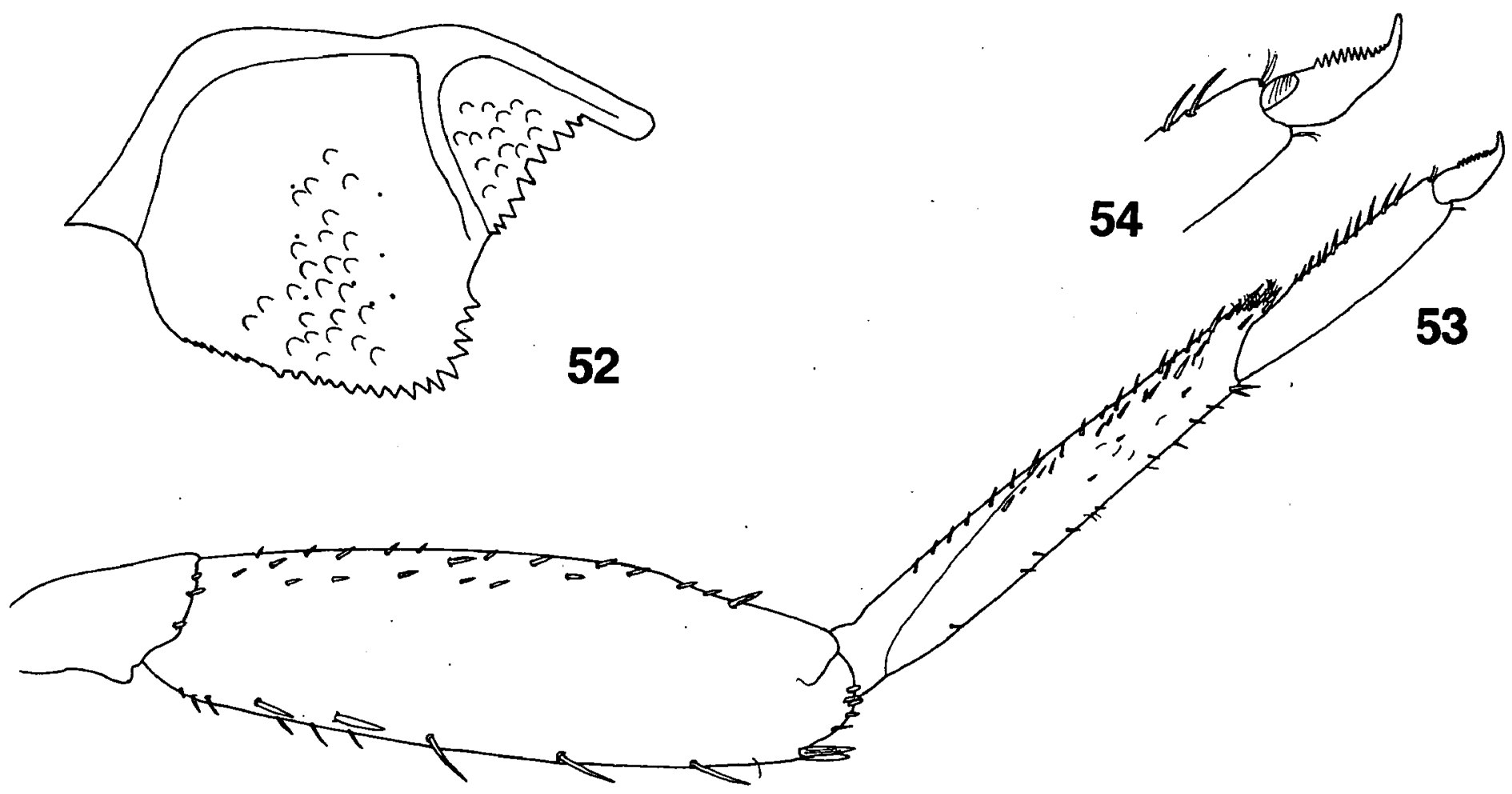

Figs. 49 to 54. Larval structures of L. punctatus sp. n. : 49 : abdomen (dorsal view). 50 : fourth gill. 51 : detail of tergum. 52 : paraproct. 53 : left foreleg. $54:$ tarsal claw.

Figs. 49 a 54 . Structures larvaires de $L$. punctatus $n$. sp. : $49:$ abdomen (vue dorsale). $50:$ quatrième branchie. $51:$ détail d'un tergite. 52 : paraprocte. $53:$ première patte gauche. $54:$ griffe tarsale. 

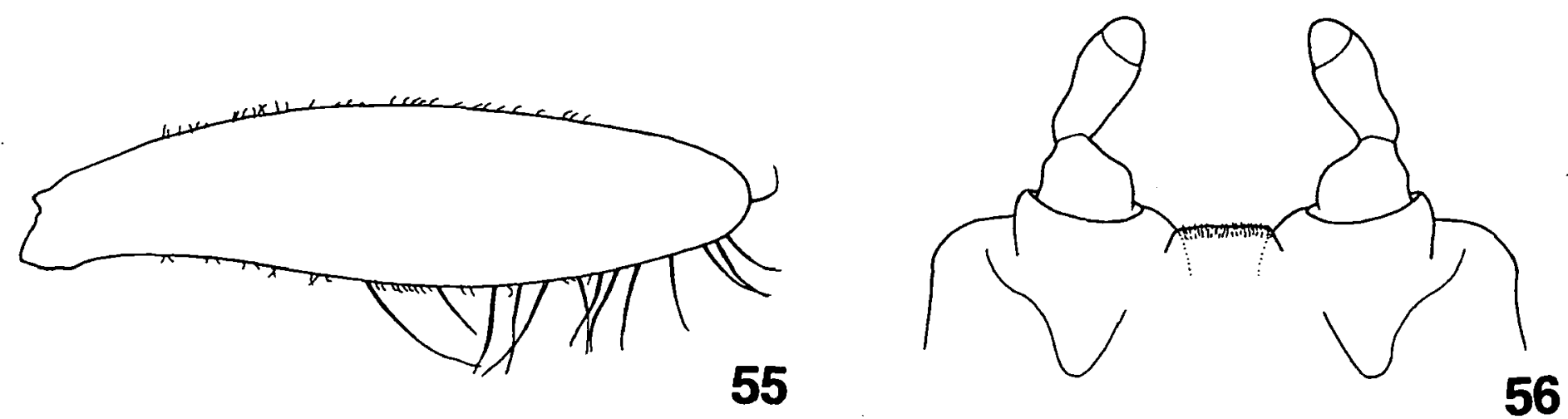

Figs. 55 to 56 . Subimaginal male structures of $L$. punctatus sp. $\mathrm{n} .: 55:$ hindwing. $56:$ genitalia.

Figs. 55 à 56 . Structures subimaginales males de L. punctatus $n$. sp. : 55 : aile antérieure. 56 : génitalia.

Madagascar, Bas. Namorona, Riv. Aff. de Namorona, Loc. $1 \mathrm{~km}$ de Vohiparara, Long. $47^{\circ} 22^{\prime} 43^{\prime \prime} \mathrm{E}$, Lat. $21^{\circ} 13^{\prime} 53^{\prime \prime} \mathrm{S}$, Alt. 1200 m, 20.4.1994. J.-M. Elouard.

One female larva 302a (on slide) and 6 larvae (P0302), Madagascar, Bas. Mangoky, Riv. Malio, Loc. Bereketa near Ranohira, Long. $45^{\circ} 08^{\prime} 28^{\prime \prime}$ E, Lat. $22^{\circ} 32^{\prime} 02^{\prime \prime} \mathrm{S}$, Alt. $645 \mathrm{~m}$, 19.4.1994. J.-M. Elouard.

One female larva 311 a (on slide), one male larva 311 b (on slide) and 11 larvae (P0311), Madagascar, Bas. Mangoky, Riv. Fenoarivo, Loc. Fenoarivo, Long. $46^{\circ} 22^{\prime} 41^{\prime \prime} \mathrm{E}$, Lat. 21 42'02" S, Alt. 725 m, 24.4.1994. J.-M. Elouard.

One male subimago with corresponding larval exuvia 339-33 (on slide), Madagascar, Bas. Mandrare, Riv. Mananara, Loc. Hazofotsy, Long. 46 35'46" E, Lat. $24^{\circ} 48^{\prime} 57^{\prime \prime} \mathrm{S}$, Alt. 100 m, 2.6.1994. J.-M. Elouard.

Two larvae (P0347), Madagascar, Bas. Mandrare, Riv. Sahandrojo, Loc. Betenina, Long. $46^{\circ} 25^{\prime} 25^{\prime \prime}$ E, Lat. $24^{\circ} 25^{\prime} 12^{\prime \prime}$ S, Alt. 325 m, 7.6.1994. J.-M. Elouard.

Two larvae (P0415), Madagascar, Bas. Mandrare, Riv. Manambolo trib. of de Mandrare, Loc. Maromby, Long. 46³4'39" E, Lat. 24²3'36" S, Alt. 345 m, 27.4.1995. J.-M. Elouard.

Two female larvae 432a and b (on slide), one male larva (on slide) and 11 larvae (P0432), Madagascar, Bas. Mangoky, Riv. Malio, Loc. Beroroha, Long. $45^{\circ} 07^{\prime} 32^{\prime \prime} \mathrm{E}$, Lat. 2140'31" S, Alt. 155 m, 29.5.1995. J.-M. Elouard.

One female larva 457b (on slide) and 5 larvae (P0457), Madagascar, Bas. Matitanana, Riv. Matitanana, Loc. Bac d'Ankarimbelo, Long. $47^{\circ} 20^{\prime} 14^{\prime \prime}$ E, Lat. $22^{\circ} 08^{\prime} 00^{\prime \prime} \mathrm{S}$, Alt. 460 m, 20.6.1995. M.R. Andriamihaja.

Two larvae (P0474), Madagascar, Bas. Manambolo, Riv. Manambolo, Loc. Andakana (bridge), Long. 46 02'00" E, Lat. $18^{\circ} 43^{\prime} 30^{\prime \prime} \mathrm{S}$, Alt. 800 m, 9.10.1995. M.R. Andriamihaja.

One female larva 604a (on slide) and 3 larvae (P0604), Madagascar, Bas. Tsiribihina, Riv. Manampanda, Loc. Antazoa, Long. $45^{\circ} 35^{\prime} 04^{\prime \prime}$ E, Lat. $20^{\circ} 21^{\prime} 40^{\prime \prime} \mathrm{S}$, Alt. $145 \mathrm{~m}$, 29.5.1996. J.-M. Elouard.
Five larvae (P0605), Madagascar, Bas. Tsiribihina, Riv. Poamay, Loc. Poamay, Long. $45^{\circ} 25^{\prime} 45^{\prime \prime}$ E, Lat. $20^{\circ} 25^{\prime} 30^{\prime \prime} \mathrm{S}$, Alt. 200 m, 30.5.1996. J.-M. Elouard.

One female larva 644b (on slide), Madagascar, Bas. Namorona, Riv. Namorona, Loc. Ranomafana, Hôtel Manja, Long. $47^{\circ} 27^{\prime} 28^{\prime \prime} \mathrm{E}$, Lat. $21^{\circ} 15^{\prime} 40^{\prime \prime} \mathrm{S}$, Alt. 725 m, 8.11.1996. J.-L. Gattolliat and C. Rochat.

One female larva 662a (on slide), Madagascar, Bas. Betsiboka, Riv. Mananta, Loc. Mananta, Long. 47 $53^{\prime} 27^{\prime \prime}$ E, Lat. 18¹9'17" S, Alt. 1160 m, 19.11.1996. J.-M. Elouard J. Legrand.

One male larva 718a (on slide) and one larva (P0718), Madagascar, Bas. Mangoro, Riv. Mangoro, Loc. Mangoro (bridge), Long. $48^{\circ} 06^{\prime} 32^{\prime \prime} \mathrm{E}$, Lat. $18^{\circ} 52^{\prime} 32^{\prime \prime} \mathrm{S}$, Alt. $840 \mathrm{~m}$, 28.4.1998. J.-M. Elouard and M. Sartori.

\section{Etymology}

The specific epithet is Latin expressing the pattern of the abdomen brown with yellow spots.

\subsection{Labiobaetis gilliesi sp. $\mathbf{n}$.}

\section{Nymph}

Maximal length, fully grown female: Body $5.3 \mathrm{~mm}$. Cerci $3.2 \mathrm{~mm}$. Terminal filament $2.1 \mathrm{~mm}$. Fully grown male: Body $5.1 \mathrm{~mm}$. Cerci $3.2 \mathrm{~mm}$. Terminal filament $2.0 \mathrm{~mm}$.

Head. Coloration almost uniformly pale brown, without vermiform marking on vertex and frons. Antennae pale yellow except scapus and pedicellus brown; pedicellus without distolateral process. Turbinate eyes honey brown.

Labrum (Fig. 57) rounded, with an anteromedial emargination, dorsally with an arc of about 10 feathered setae, reaching margin, abundant long and thin setae scattered over surface; distal margin bordered with thin simple setae; ventrally with two stout, minute setae and a disto-medial arc of very thin setae. 

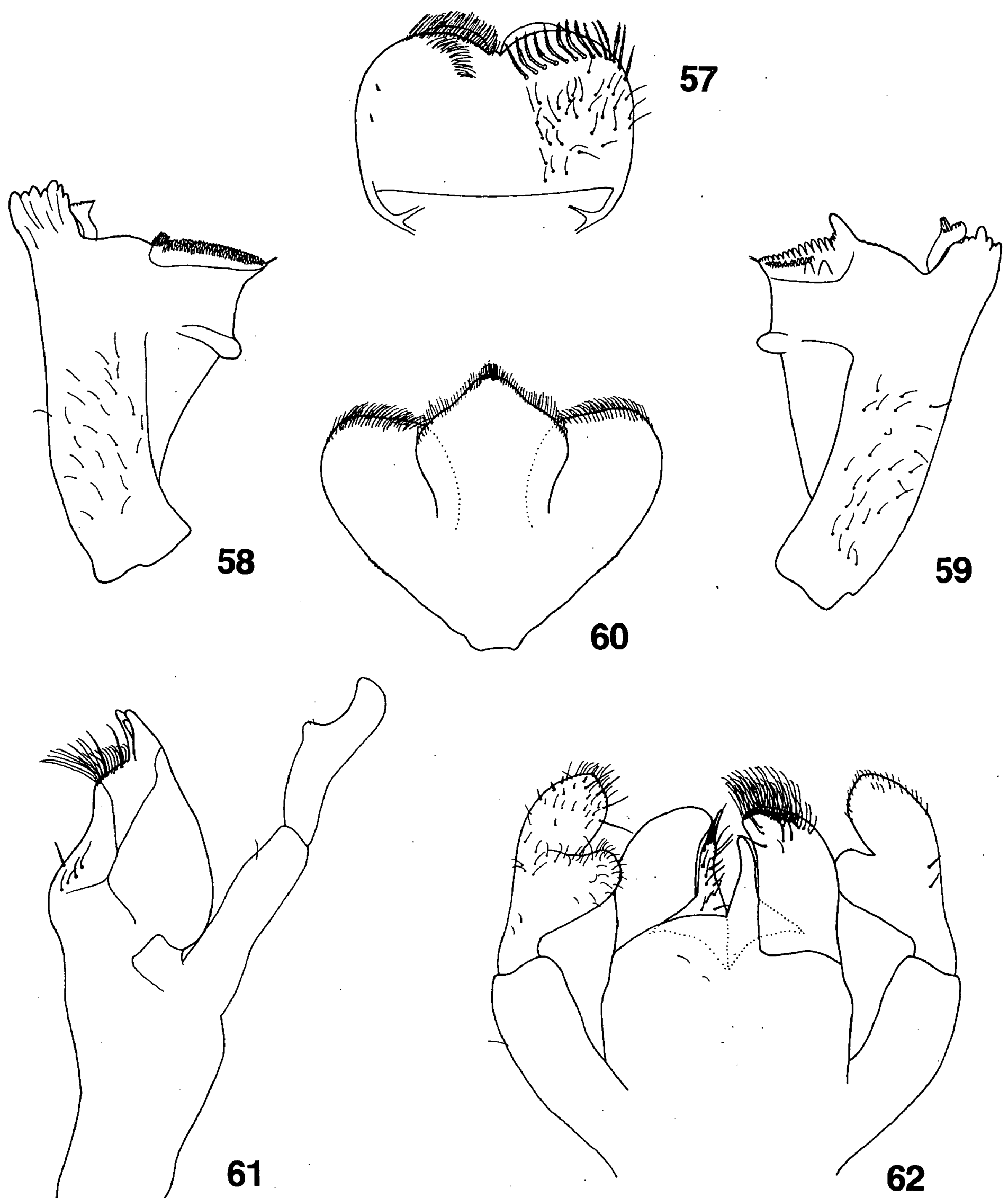

Figs. 57 to 62 . Larval structures of $L$. gilliesi sp. n. : $57:$ labrum (left : ventral; right : dorsal). $58:$ right mandible. $59:$ left mandible. 60 : hypopharynx. 61 : left maxilla. 62 : labium.

Figs. 57 à 62. Structures larvaires de L. gilliesi n. sp. : 57 : labre (gauche : ventral; droite : dorsal). $58:$ mandibule droite. 59 : mandibule gauche. 60 : hypopharynx. 61 : maxille gauche. 62 : labium. 

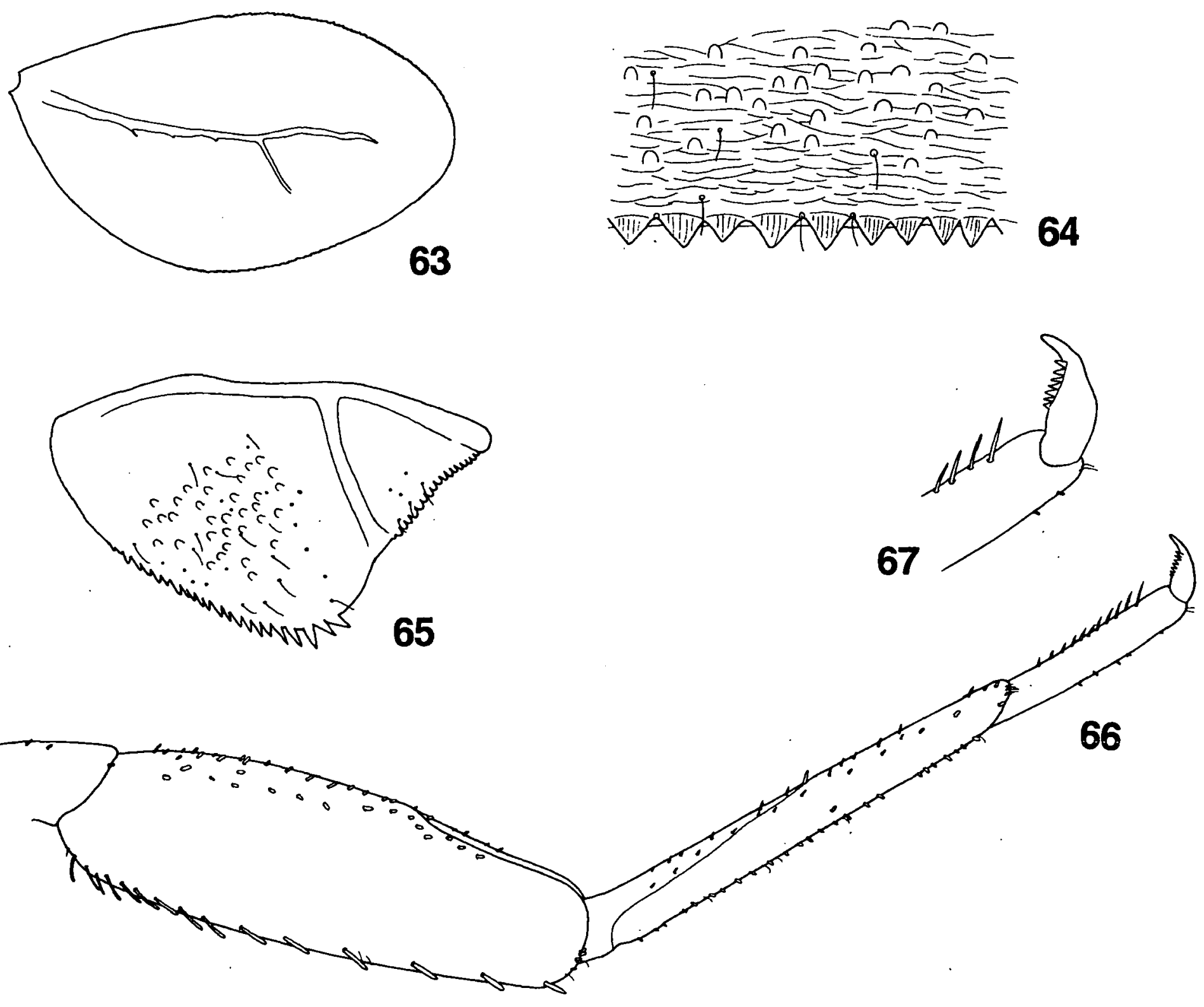

Figs. 63 to 67 . Larval structures of $L$. gilliesi sp. n. : 63 : fourth gill. 64 : detail of tergum. 65 : paraproct. $66:$ left foreleg. 67 : tarsal claw.

Figs. 63 à 67. Structures larvaires de L. gilliesi n. sp. : 63 : quatrième branchie. 64 : détail d'un tergite. 65 : paraprocte. 66 : première patte gauche. 67 : griffe tarsale.

Right mandible (Fig. 58), stout prostheca apically with rounded denticules; margin between prostheca and mola crenate, without hump and without setae; tuft of setae at apex of mola reduced to two small setae; basal half with long and thin setae dorsally.

Left mandible (Fig. 59), stout prostheca apically with denticules and a comb-shape structure; margin between prostheca and mola crenate, almost straight, without hump and without setae; tuft of setae at apex of mola reduced to two setae; basal half with long and thin setae dorsally.

Hypopharynx (Fig. 60), superlingua with a median projection, covered with black stout setae.

Maxillae (Fig. 61) with 4 teeth, none of them opposed to others; row of setae ending with eight long se- tae, two spine-like setae in middle of row; row of 4 setae at basis of galea; one single of seta perpendicular to margin of galea; palp 2-segmented, longer than galealacinia, segment 2 with a pronounced distomedial concavity.

Labium (Fig. 62) with glossae clearly shorter than paraglossae; glossae slender, inner margin with long setae, ventrally with stout setae; paraglossae stout, apically rounded, with 3 to 4 rows of pectinate setae, row of 3 setae parallel to inner margin. Mentum with 2 thin setae apically. Labial palp 3-segmented slender; first segment elongated with few thin setae, subequal to second and third combined; second segment with a well-developed rounded thumb-like distomedial projection, distal margin concave, with few thin setae, 
two small setae dorsally; third segment subconical and subsymmetrical, slightly pointed, covered with short thin setae and few stouter ones, longer setae on inner margin.

Thorax. Thorax with medially a pale yellow longitudinal stripe surrounded by a symmetrical brown stripe.

Hindwing pads present.

Legs uniformly light yellow.

Forelegs (Fig. 66), coxa with a single seta on distal margin.

Femora with a row of 15 blunt setae dorsally; apicodorsal patch formed by a single seta similar to those of dorsal margin; apex with 4 blunt setae; ventral margin with numerous small blunt setae.

Tibiae dorsally with a row of small blunt setae; apically with a single small blunt seta; ventrally with few pointed setae, quite rare apically; tibio-patellar suture present.

Tarsi with few minute setae dorsally; ventral margin with a row of pointed setae increasing in length toward apex; tarsal claws (Fig. 67) with one row of about 7 teeth; subapical setae absent.

Second and third legs similar to foreleg.

Abdomen. Coloration brown with medially a pale yellow longitudinal stripe.

Terga (Fig. 64) shagreen with scale bases and few setae; posterior margin with triangular spination, slightly broader than long.

Sterna pale yellow, except laterally brown, shagreen with scale bases and setae; smooth posterior margin without spines.

Gills (Fig. 63) present on abdominal segments 1 to 7 , tracheation brown almost without ramification, serrated distally, gill 1 clearly reduced.

Paraproct (Fig. 65) with about 30 scale bases and few setae, margin with about 25 pointed spines; postero-lateral extension without scale base, rounded spines along margin.

Cerci coloration yellowish brown, except median segments brown giving a banded appearance.

\section{Adult}

Unknown

\section{Material examined}

\section{Holotype}

- Male Larya (P0869) Madagascar, Bas. Mangoro, Riv. Mangoro, Loc. Mangoro (bridge), Long. $48^{\circ} 06^{\prime} 32^{\prime \prime} \mathrm{E}$, Lat. $18^{\circ} 52^{\prime} 32^{\prime \prime}$ S, Alt. $840 \mathrm{~m}, 12.4 .1999$. J.-L. Gattolliat and N. Raberiaka.

\section{Paratypes}

One female larva 869a (on slide), one male larva 869 b (on slide) and 2 larvae (P0869), same data as holotype.

Three larvae (P0112), same locality as holotype, 15.11.1991. J.-M. Elouard.

Four larvae (P0115), same locality as holotype, 10.3.1993. J.-M. Elouard.

One female larva 50a (on slide) and 3 larvae (P0050), Madagascar, Bas. Betsiboka, trib. of Antroby, Long. $47^{\circ} 02^{\prime} 32^{\prime \prime}$ E, Lat. 18¹1'38" S, Alt. 1070 m, 19.4.1991. J.-M. Elouard.

One male larva 577a (on slide), one female larva 577b (on slide) and 7 larvae (P0577), Madagascar, Bas. Betsiboka, Riv. Mamokomita, Loc. Manjakavaradrano, Long. 4659'20" E, Lat. 1740'55" S, Alt. 675 m, 3.4.1996. J.-M. Elouard.

One female larva 604a (on slide) and 3 larvae (P0604), Madagascar, Bas. Tsiribihina, Riv. Manampanda, Loc. Anta-

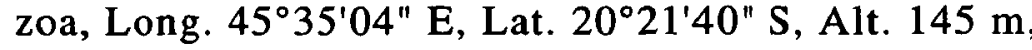
29.5.1996. J.-M. Elouard.

Five larvae (P0605), Madagascar, Bas. Tsiribihina, Riv. Poamay, Loc. Poamay, Long. $45^{\circ} 25^{\prime} 45^{\prime \prime} \mathrm{E}$, Lat. $20^{\circ} 25^{\prime} 30^{\prime \prime} \mathrm{S}$, Alt. $200 \mathrm{~m}, 30.5 .1996$. J.-M. Elouard.

\section{Etymology}

This species is dedicated to the late distinguished Ephemeropterist M.T. Gillies.

\subsection{Labiobaetis fabulosus Lugo-Ortiz and McCaf- ferty}

\section{Nymph}

Original description see Lugo-Ortiz \& McCafferty 1997 and figure 68.

\section{Adult}

Length, male and female imagoes: Body $4.7-7.0$ $\mathrm{mm}$. Forewing $4.8-7.6 \mathrm{~mm}$. Hindwing $1.0-1.2 \mathrm{~mm}$.

Head. Brown to dark brown. Turbinate eyes dark purple brown. Eyes black. Antennae with basis of scapus and pedicellus dark brown, flagellum light yellow with a brown stripe.

Thorax. Forewings 4.8 to $7.6 \mathrm{~mm}$, amber brown with golden brown veins; double intercalary veins between longitudinal veins; pterostigma with 10 to 14 cross-veins, some of them forked or fused (Fig. 69).

Hindwings amber brown, narrow, crescent-shaped, with two longitudinal veins, without costal process (Fig. 70).

Legs uniformly light yellow.

Terga brown, apically darker; terga 7 to 9 dark brown.

Male genitalia (Fig. 71) with three-segmented gonopods, first and second segments almost fused, first seg- 


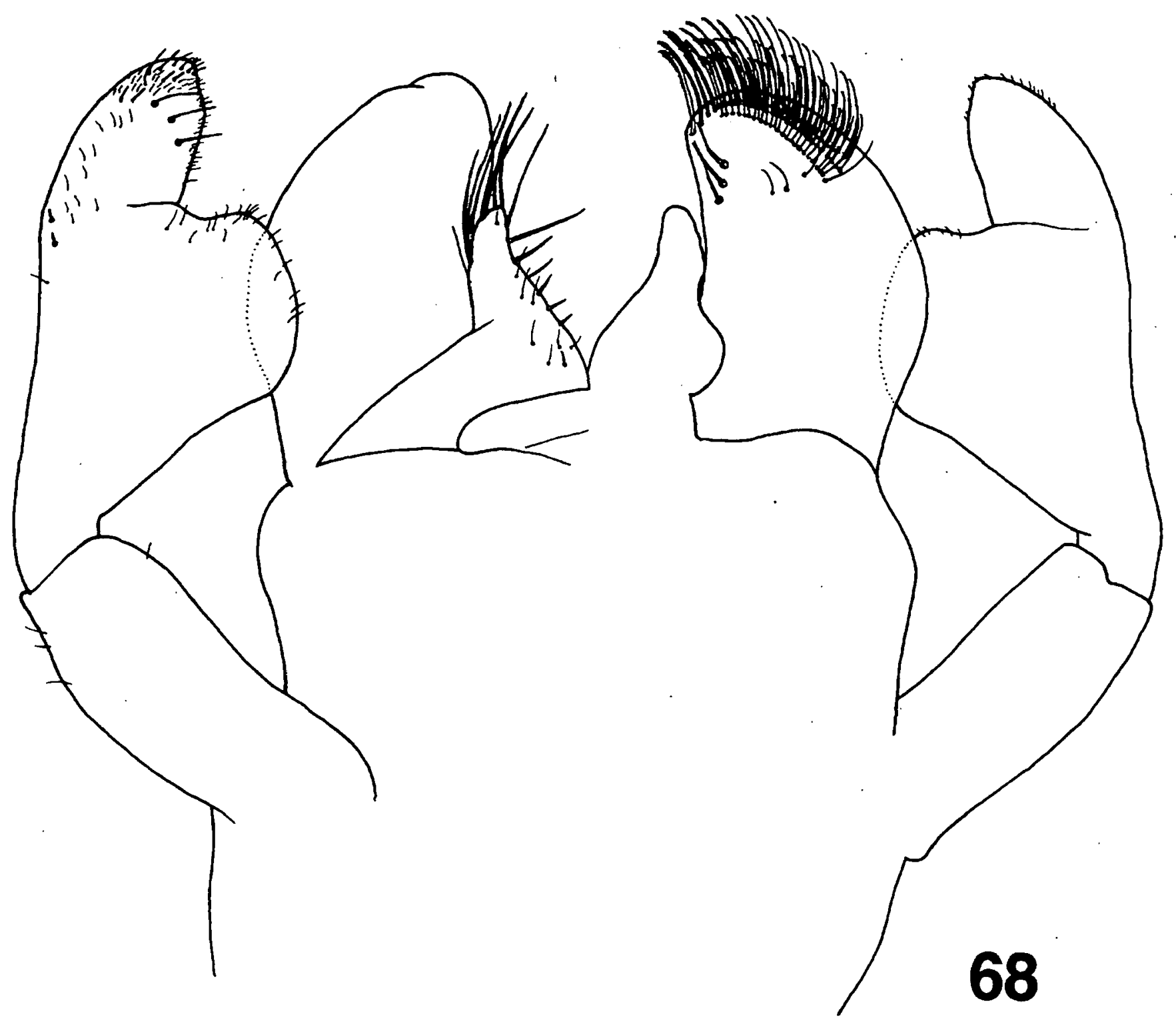

Fig. 68. Larval structures of $L$. fabulosus Lugo-Ortiz and McCafferty : labium.

Fig. 68. Structures larvaires de L. fabulosus Lugo-Ortiz et McCafferty : labium.

ment without apophysis, abundant thin setae present on inner margin of second segment, third segment truncated; poorly-developed sclerotized process between forceps, as broad as distance between forceps, apically rounded.

\section{Material examined}

One male imago with corresponding larval and subimaginal exuvia 200a (on slide), Madagascar, Bas. Antongombato, Riv. Makis, Loc. Camp base WWF, Long. $49^{\circ} 10^{\prime} 09^{\prime \prime}$ E, Lat. $12^{\circ} 31^{\prime} 40^{\prime \prime}$ S, Alt. $1075 \mathrm{~m}, 2.4 .1994$. J.-M. Elouard and M. Sartori.

One female larva 213e (on slide) and 11 larvae (P0213), Madagascar, Bas. Namorona, Riv. Tamara, Loc. $4 \mathrm{~km}$ from Ranomafana, Long. $47^{\circ} 25^{\prime} 37^{\prime \prime} \mathrm{E}$, Lat. $21^{\circ} 14^{\prime} 45^{\prime \prime} \mathrm{S}$, Alt. $850 \mathrm{~m}, 17.4 .1994$. J.-M. Elouard and M. Sartori.

Two male imagoes 226b and 226c and one female imago $226 \mathrm{~d}$ with corresponding larval and subimaginal exuvia (on slide), Madagascar, Bas. Namorona, Riv. Namorona, Loc. road Ifanadiana-Tolongoina, Long. $47^{\circ} 35^{\prime} 55^{\prime \prime} \mathrm{E}$, Lat. $21^{\circ} 22^{\prime} 40^{\prime \prime}$ S, Alt. $500 \mathrm{~m}, 22.4 .1994$. J.-M. Elouard and M. Sartori.

One male imago 238a and three larvae (P0238), Madagascar, Bas. Namorona, tributary of Namorona, Loc. Tsarafidy, Long. $47^{\circ} 16^{\prime} 03^{\prime \prime} \mathrm{E}$, Lat. $21^{\circ} 13^{\prime} 02^{\prime \prime} \mathrm{S}$, Alt. $1260 \mathrm{~m}$, 27.4.1994. J.-M. Elouard.

One male imago with corresponding larval and subimaginal exuvia 330-4 (on slide), Madagascar, Bas. Mandrare, Riv. Imonty, Loc. Imonty, Long. $46^{\circ} 41^{\prime} 25^{\prime \prime} \mathrm{E}$, Lat. $24^{\circ} 48^{\prime} 51^{\prime \prime}$ S, Alt. 175 m, 21.5.1994. J.-M. Elouard.

One male larva 526a (on slide) and two larvae (P0526), Madagascar, Bas. Manampanihy, Riv. Manampanihy, Loc. Enosiary, Long. 46 49'19" E, Lat. 24 40'37" S, Alt. 100 m, 21.11.1995. J.-M. Elouard and T. Pilaka.

Two male imagoes 598-2 and 598-3 (on slide), Madagascar, Bas. Tsiribihina, Ivato river, Ivato, Long. $47^{\circ} 10^{\prime} 30^{\prime \prime} \mathrm{E}$, Lat. $20^{\circ} 39^{\prime} 40^{\prime \prime}$ S, Alt. $1500 \mathrm{~m}, 23.5 .1996$. J.-M. Elouard.

One male imago 654-1 (on slide) and one male imago (P0654), Madagascar, Bas. Tsiribihina, Loc. Ilaka-Afovoa- 


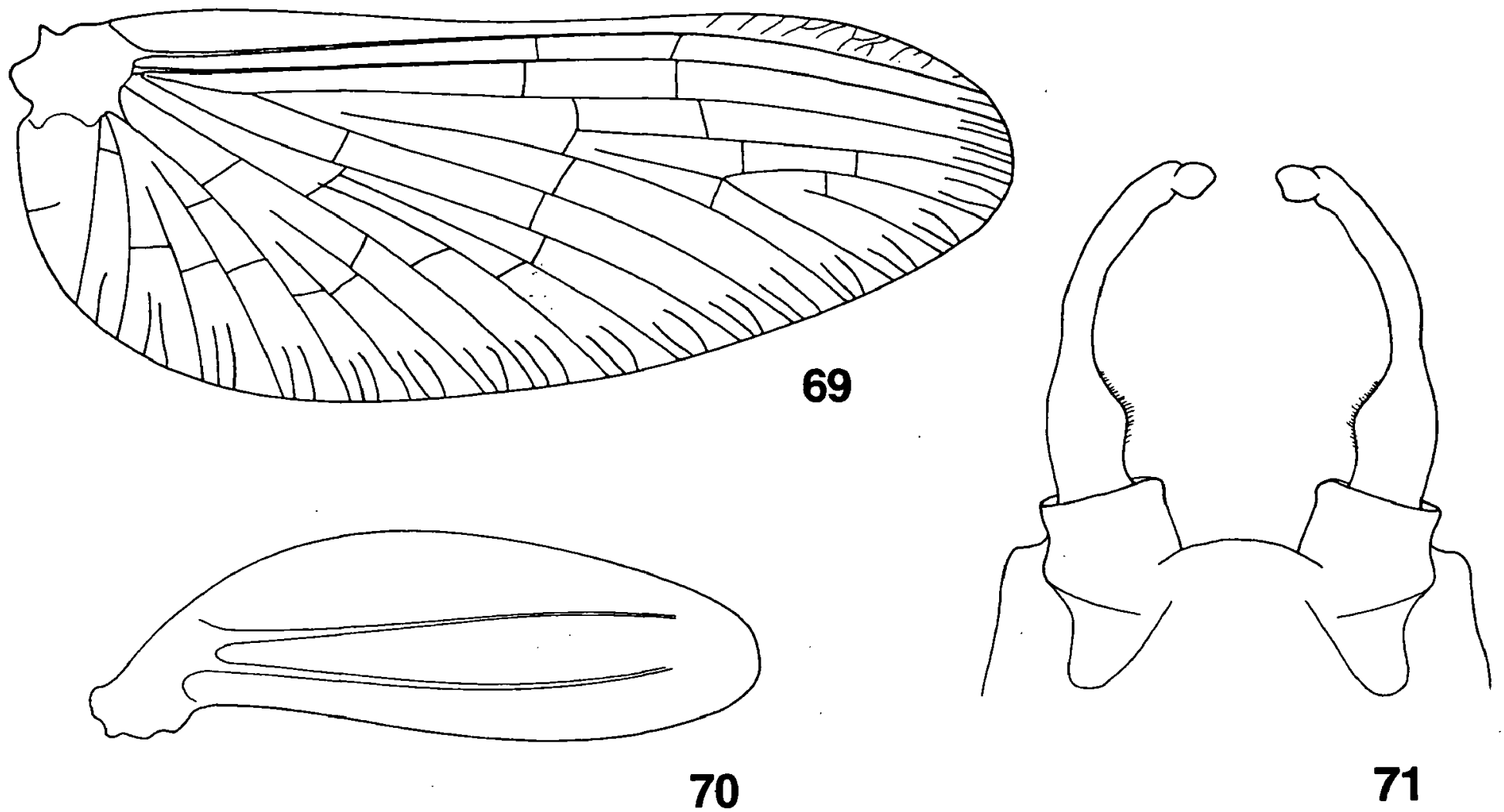

Figs. 69 to 71 . Imaginal male structures of L. fabulosus Lugo-Ortiz and McCafferty : 69 : forewing. 70 : hindwing. 71 : genitalia.

Figs. 69 à 71. Structures imaginales males de L. fabulosus Lugo-Ortiz et McCafferty : 69 : aile antérieure. 70 : aile postérieure. 71 : génitalia.

ny, Long. $47^{\circ} 10^{\prime} 35^{\prime \prime} \mathrm{E}$, Lat. $20^{\circ} 22^{\prime} 00^{\prime \prime} \mathrm{S}$, Alt. $1450 \mathrm{~m}$, 14.11.1996. J.-L. Gattolliat.

One male larva 776a (on slide) and three larvae (P0776), Bas. Ivondro, Riv. Manambolo, Loc. Ambodibonara, near

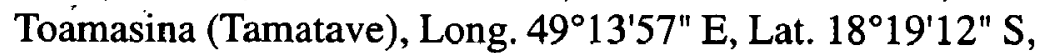
Alt. 15 m, 29.10.1998. J.-M. Elouard, J. Legrand and Z. Rabeantoandro.

One male larva 822a and forty-five larvae (P0822), Madagascar, Antongombato Bas., Makis Riv., Loc. 100m downstream of the Great Waterfall (Montagne d'Ambre), Long. $49^{\circ} 10^{\prime} 14^{\prime \prime}$ E, Lat. 12²9'17" S, Alt. 675 m, 24.3.1999. J.-L. Gattolliat and Z. Rabeantoandro.

One female larva 833a (on slide) and twenty-five larvae (P0833), Madagascar, Bas. Sahankazo, Riv. Antsandrangotika, Long. $49^{\circ} 23^{\prime} 46^{\prime \prime}$ E, Lat. $12^{\circ} 28^{\prime} 40^{\prime \prime} \mathrm{S}$, Alt. $50 \mathrm{~m}$, 29.3.1999. J.-L. Gattolliat and Z. Rabeantoandro.

Two female larvae $860 \mathrm{~d}$ and $860 \mathrm{e}$ (on slide) and thirty larvae (P0860), Madagascar, Bas. Rianila, Riv. Sahatandra, Loc. Ambodiriana, near Ambalafary road to Lakato. Long. $48^{\circ} 20^{\prime} 19^{\prime \prime}$ E, Lat. $19^{\circ} 01^{\prime} 30$ S, Alt. 980 m. 7.4.1999. J.-L. Gattolliat and N. Raberiaka.

\section{Discussion}

McCafferty \& Waltz (1995) raised Labiobaetis to the generic rank and attributed to it most species of the Baetis propinquus, B. atrebatinus and B. molawinensis species group. Despite the dubious status of the confusing genus Pseudocloeon, all the species previously assigned to Labiobaetis were recently reassigned to Pseudocloeon (Lugo-Ortiz et al. 1999)

A great uncertainty still exists in the taxonomic situation of the genus Pseudocloeon. This genus was established by Klapálek for four imagoes from Java, the nymphal stage remaining unknown. He characterized it by paired marginal intercalaries and absence of hindwings. Numerous species with this combination of characters were consequently classified as Pseudocloeon. These attributions are considered as provisional (Müller-Liebenau 1981): the loss of hindwing is a homoplasy associated with size reduction that have occurred in many diverse lineages of baetids (McCafferty \& Waltz 1995). The species assigned to Pseudocloeon were consequently polyphyletic. During the last decade, most species have been reassigned to other genera of the Baetis complex (Lugo-Ortiz et al. 1999).

As previously stated by Waltz \& McCafferty (1985, 1987), the knowledge of the larval stage is essential to understanding and defining generic limits within the Baetidae and especially within the Baetis complex. Most genera included in the Baetis complex present important apomorphies at the larval stages, but the ge- 
neric attributes remain unclear at the imaginal stage. Consequently the concept of Pseudocloeon will remain problematic until the larval-imaginal correspondence is known. The attempt to describe the larval stage based on North American species is subject to caution as far as the assignment of these species to Pseudocloeon is generally assumed but not proven (LugoOrtiz et al. 1999). The only reliable feature at the imaginal stage that allows the distinguishment of Pseudocloeon sensu Lugo-Ortiz et al. (1999) from other genera of the Baetis complex is the sclerotized median platelike process at the base of the male genitalia. In the redescription of the type species, Waltz \& McCafferty (1985) indicate that this process is absent in P. kraepelini. Moreover McCafferty \& Waltz (1995) consider this platelike process as plesiomorphic. Consequently, this plate cannot be deemed as an apomorphy.

Lugo-Ortiz et al. (1999) indicate that the male genitalia of $P$. kraepelini are equivalent to those of Labiobaetis and conclude that Pseudocloeon encompasses the present concept of Labiobaetis. However, it can be seen that this conclusion is only based on the speculation by Waltz \& McCafferty $(1985,1987)$ who only suggested that $P$. kraepelini was probably congeneric with the species of the $B$. atrebatinus group, but never showed that they were equivalent. Moreover, it must be noticed that no apomorphical feature at the imaginal stage allows the separation of Pseudocloeon from Baetis. Other genera in the Baetis complex are also present in Indonesia (near the type locality of Pseudocloeon) but the imaginal stages also remain unknown.

The wisest solution is to restrict the concept of Pseudocloeon to the type-species $P$. kraepelini, as proposed by Waltz \& McCafferty (1985 and 1987).

Labiobaetis sensu McCafferty \& Waltz (1995) is rather heterogeneous. Important apomorphies such as the antennal process, the excavation of the maxillary segment 2, the enlargement of the labial palp segment 2 or even the presence of villopores are sometimes absent or poorly developed (Waltz \& McCafferty 1985 , 1987, Lugo-Ortiz et al. 1999).

Consequently, I attribute the six new species described herein to the genus Labiobaetis. However, it will be premature to reassign to Labiobaetis all the species previously assign to Pseudocloeon until a global revision of these genera is led, based on material from throughout their range.

The eight Malagasy species of Labiobaetis present the main generic features such as maxillary palp segment 2 distomedially excavated, labial palp segment 2 with a distomedial projection, tarsal claws with one row of teeth, forewings with double intercalary vein. However, the villopores and the distolateral process of the antennal scape are absent or at least poorly developed.

These Malagasy species can be easily distinguished by the shape and the degree of development of labial palp segment 2 , the shape of the maxillary palp excavation, the shape of the paraglossae, the shape and the setation of the labrum, the number and the degree of development of the pointed setae of the dorsal femoral margin, the presence or absence of hindwings and the number of gills.

Labiobaetis fabulosus is the most specialised species; its hypognathous mouthparts and the high modification and reduction of the glossae distinguish it from all the species of Labiobaetis. The genus Glossidion Lugo-Ortiz and McCafferty presents similar adaptation of the glossae but it differs by the shape of the labrum, the maxillary palp segment 2 being bulbous without excavation and the labial palp lacks the distomedial projection (Lugo-Ortiz \& McCafferty 1998).

Labiobaetis vulgaris appears closely related to $L$. plumbago Lugo-Ortiz and McCafferty, and also to the African species $L$. vinosus (Barnard). They all possess six pairs of gills, no hindwings and have high similarities of the mouthparts. Labiobaetis plumbago differs from $L$. vulgaris and $L$. vinosus and all other Afrotropical species of Labiobaetis by the distal margin of terga with elongated furrows and the margin of posterolateral extension of the paraproct with furrows. Despite the huge number of samples made by the LRSAE team including at the paratype locality, we never found any specimens of $L$. plumbago.

Labiobaetis dambrensis and L. nigrocercus present few differences (shape of the segment 2 of the maxillary palp, setae of the dorsal margin of the femora, number of gills), but the simultaneous presence of the two species at the same localities is important, suggesting these differences are not due to variations among different populations.

Labiobaetis longicercus has 7 pairs of gills and no hindwings. This is rather unusual: most species that do not possess hind wing have only 6 pairs of gills (LugoOrtiz et al., 1999). The only other exception is $L$. difficile (Müller-Liebenau) (Müller-Liebenau 1984). Labiobaetis gilliesi and $L$. punctatus are both typical of the genus Labiobaetis.

The imagoes of only five of the eight species are known. The venation of their forewings is constant. The hindwings, when present, are similar except for the coloration in L. fabulosus. The genitalia are also similar; only $L$. vulgaris possesses a median platelike 
process which is reduced and rounded, and the third segment of the genitalia of $L$. fabulosus is truncated instead of globular as in the other Malagasy species.

\section{Keys to the Malagasy species of Labiobaetis}

\section{Key to nymphs}

1 - Hindwings pads present 2

- Hindwings pads absent

2 - Mouthparts hypognathous; glossae strongly reduced, less than the third of paraglossae

Labiobaetis fabulosus Lugo-Ortiz \& McCafferty - Mouthparts arranged as usual; glossae not reduced, slightly shorter than paraglossae

3 - Second segment of maxillary palp greatly enlarged at the basis

- Second segment of maxillary palp not greatly enlarged at the basis

$4-7$ pairs of gills, cerci with a black stripe

L. nigrocercus $\mathrm{sp} . \mathrm{n}$.

- 6 pairs of gills, cerci without a black stripe

L. dambrensis sp. $\mathrm{n}$.

5 - Abdomen uniformly brown, with a longitudinal pale stripe; dorsal margin of femora with more than 10 blunt setae; ventral margin of tibiae with few setae; second segment of labial palp with a thumb-like distomedial rounded projection

L. gilliesi sp. $\mathrm{n}$.

- Abdomen dark brown, with 4 yellow spot on each tergum; dorsal margin of femora with less than 7 pointed setae; ventral margin of tibiae with numerous setae especially apically; second segment of labial palp with a triangular disto-medial projection

L. punctatus $\mathrm{sp} . \mathrm{n}$.

6 - Cerci at least $1.5 \mathrm{x}$ longer than the body; 7 pairs of gills; disto-medial projection of labial palp poorly developed; right and left mandibles without a hump between prostheca and mola

L. longicercus $\mathrm{sp} . \mathrm{n}$.

- Cerci shorter than the body; 6 pairs of gills; disto-medial projection of labial palp well-developed; one mandible with a hump between prostheca and mola

7 - Left mandible with a hump between prostheca and mola; labrum broad, distal margin of terga with elongate furrows and small blunt subtriangular spination, margin of postero-lateral extension of paraprocts with furrows

L. plumbago Lugo-Ortiz \& McCafferty

- Right mandible with a hump between prostheca and mola; labrum narrow and rounded; distal margin of terga with triangular pointed spination without furrows, margin of postero-lateral extension of paraprocts with blunt spination but without furrows

L. vulgaris sp. $\mathrm{n}$.

\section{Key to male imagoes}

1 - Hindwings pads present 2

- Hindwings pads absent 3

2 - Forewings and hindwings amber brown; third segment of genitalia convex apically

L. fabulosus Lugo-Ortiz \& McCafferty

- Forewings and hindwings hyaline; third segment of genitalia rounded

L. dambrensis sp. n.*

L. punctatus sp. $\mathrm{n}$ *

3 - Genitalia with median platelike process apically flattened

L. longicercus sp. $\mathrm{n}$.

- Genitalia with median platelike process reduced and subrounded

L. vulgaris sp. $\mathrm{n}$.

* in the type-material, the small size of the imagoes of $L$. punctatus allows their differentiation from $L$. dambrensis

\section{References}

Lugo-Ortiz C.R. \& McCafferty W.P. 1997. - Labiobaetis (Ephemeroptera: Baetidae) from the Afrotropical region. Afr. Entomol., 5 : 241-260.

Lugo-Ortiz C.R. \& McCafferty W.P. 1998. - A new Baetis-complex genus (Ephemeroptera: Baetidae) from the Afrotropical region. Afr. Entomol., 6 (2) : 297-301.

Lugo-Ortiz C.R., McCafferty W.P. \& Waltz R.D. - 1999. Definition and reorganisation of the genus Pseudocloeon (Ephemeroptera : Baetidae) with new species descriptions and combinations Trans. Am. Entomol. Soc., 125 (1-37) : 208-211.

McCafferty W.P. \& Waltz R.D. 1995. - Labiobaetis (Ephemeroptera : Baetidae): new status, new North American species and related new genus. Ent. news, 106 (1) : 19-28.

Müller-Liebenau I. 1981. - Review of the original material of the baetid genera Baetis and Pseudocloeon from the Sunda Islands and the Philippines described by G. Ulmer, with some general remarks (Insecta: Ephemeroptera). Mitt. Hamburg Zool. Mus. Inst., $78: 197-208$.

Müller-Liebenau I. 1984. - New genera and species of the family Baetidae from West-Malaysia (River Gombak). Spixiana, 7 : 253-284.

Novikova E.A. \& Kluge N.Y. 1987. - Systematics of the genus Baetis (Ephemeroptera, Baetidae) with description of a new species from Middle Asia. Vestn. Zool., (4) : 8-19.

Waltz R.D. \& McCafferty W.P. 1985. - Redescription and new lectotype designation for the type species of Pseudocloeon, P. krae pelini Klapálek (Ephemeroptera: Baetidae). Proc. Entomol. Soc. Wash., 87 (4) : 800-804

Waltz R.D. \& McCafferty W.P. 1987. - Systematics of Pseudocloeon, Acentrella, Baetiella and Liebebiella (Ephemeroptera: Baetidae). J.N.Y. Entomol. Soc., 87 : 553-568. 